\title{
A Review of Strongyloides spp. Environmental Sources Worldwide
}

\author{
Mae A. F. White *(i), Harriet Whiley ${ }^{(\mathbb{D}}$ and Kirstin E. Ross ${ }^{(1)}$ \\ Environmental Health, College of Science and Engineering, Flinders University, GPO Box 2100, \\ Adelaide 5001, Australia \\ * Correspondence: mae.white@flinders.edu.au; Tel.: +61-8-7221-8585
}

Received: 4 June 2019; Accepted: 23 June 2019; Published: 27 June 2019

\begin{abstract}
Strongyloides spp. are parasitic nematodes that are transmitted through the environment and are capable of causing disease. These nematodes affect an estimated 3-300 million humans worldwide. Identifying the environmental reservoirs of Strongyloides spp. is essential for the development of appropriate control strategies. This systematic literature review examined all published studies that identified Strongyloides stercoralis, Strongyloides fuelleborni, Strongyloides fuelleborni kellyi, and Strongyloides spp. from an environmental source. Most studies detected the nematode from dog and primate fecal samples. Other environmental sources identified were ruminants, cats, rodents, insects, water, soil, as well as fruit and vegetables. Most studies used microscopy-based identification techniques; however, several employed molecular-based techniques, which have become increasingly popular for the detection of Strongyloides spp. A limitation identified was a lack of studies that comprehensively screened all potential environmental samples in a region. Future research should undertake this holistic screening process to identify which environmental reservoirs pose the greatest significance to human health. Potential controls can be identified through the identification of environmental sources. Understanding where Strongyloides spp. is commonly found within the environment of endemic areas will inform environmental control strategies to reduce this neglected disease.
\end{abstract}

Keywords: Strongyloides spp.; Strongyloides stercoralis; Strongyloides fuelleborni; strongyloidiasis; environmental reservoirs

\section{Introduction}

Strongyloidiasis is a disease caused by parasitic nematodes of the genus Strongyloides. Within this genus, three species, Strongyloides stercoralis, Strongyloides fuelleborni, and Strongyloides fuelleborni kellyi are known to parasitize humans [1,2].

S. stercoralis, S. fuelleborni, and S. fuelleborni kellyi are capable of autoinfecting the host. This occurs after adult female parthenogenic nematodes within the infected human shed eggs. These eggs develop to larvae that are passed within the stool. A certain number burrow through the wall of the large intestine, thereby reinfecting the body. Infected individuals can have a low-level undetected infection for many years [3]. When this auto-infective life cycle becomes uncontrolled in immunocompromised, young, and elderly patients, a disseminated infection can develop. Disseminated infection occurs when the parasite travels throughout the body. This can result in sepsis, bacterial meningitis, or gastrointestinal hemorrhage [4]. The mortality rate from a disseminated infection and its comorbidities is estimated to be $80 \%$ [3]. The larvae that are passed within the stool are then capable of completing a free-living cycle, in which they molt twice to develop into filariform larvae. These infective filariform larvae are capable of then reinfecting humans, where they can be involved with the autoinfection cycle again [5]. 
Both S. stercoralis and S. fuelleborni are able to complete their life cycle within animals such as canids, primates, and insects. Animal species-specific strains of $S$. stercoralis unable to infect humans have been identified [6]. This ability for the nematode to reproduce within other animals indicates that all infected animals' feces may pose an infection threat to humans.

After excretion in the stool, larvae can survive and reproduce within the environment, and environmental sources contaminated with larvae can cause reinfection. Although Strongyloides spp. are classified as soil-transmitted helminths, locations that harbor Strongyloides spp. within the environment, with the exception of soil, have not been investigated holistically [7,8]. By reviewing and collating all reported environmental sources of S. stercoralis, S. fuelleborni, and S. fuelleborni kellyi, environmental interventions can be implemented.

We need a better understanding of the environmental sources of Strongyloides spp.; resistance to the current anthelminthic drugs has been observed in other Strongyloides spp. [9]. Both environmental and clinical control of Strongyloides spp. is essential [10]. The aim of this review is to identify all research reporting S. stercoralis, S. fuelleborni, S. fuelleborni kellyi, and Strongyloides spp. within environmental sources worldwide.

\section{Results}

One thousand two hundred and twenty-two papers were retrieved from SCOPUS and Web of Science using the search terms identified as suitable, as seen in Table 1, with 174 articles identified as eligible for inclusion.

S. stercoralis was identified in 35\% of all studies and S. fuelleborni in $10 \%$ of all studies; both S. stercoralis and S. fuelleborni were identified in 1\% of all studies. S. fuelleborni and Strongyloides spp. were identified in $0.5 \%$ of studies, and genus-level identification was identififed in $55 \%$ of all studies, as seen in Table A1. S. fuelleborni kellyi was not identified within any papers.

The most commonly identified reports of Strongyloides spp. were within primates ( $26 \%$ of all published works), and dogs (14\% of all published works), as seen in Table A1. Other animals identified as environmental sources included cats, ruminants, rodents, and insects. Water, soil, as well as fruit and vegetables were all also identified as containing Strongyloides spp.

Fifty percent of all studies identifying Strongyloides spp. within primate populations identified the larvae to genus level only. S. fuelleborni was the next most frequently identified species at $40 \%$. Parasitic infections were identified more frequently in terrestrial primates than arboreal primates [11,12]. Most studies $(80 \%)$ employed microscopy, as seen in Table A1. Proximity to human populations and increased interaction with human populations was also frequently reported in infected populations $[13,14]$. Captive primates treated with anthelmintic drugs were also reported as carriers of Strongyloides spp. [15]. Sample size ranged from 7 to 3349 , and prevalence within primate studies ranged from $<1 \%$ to $100 \%$, as seen in Table A1.

Domestic and stray dogs were the second most commonly identified source. Fourteen percent of all studies identifying Strongyloides spp. were within dogs, with sample sizes ranging from 35 to 879 and prevalence ranged from $<1 \%$ to $45 \%$, as seen in Table A1.

All studies reporting incidences of Strongyloides spp. within ruminant farming animals only identified Strongyloides to the genus level, as seen in Table A1.

Studies identifying rodents as a source of Strongyloides spp. accounted for $5 \%$ of the published works. Studies identified Rattus rattus, Rattus norvegicus, Mus musculus, Dasyprocta, and Hydrochoerus hydrochaeris as carriers of Strongyloides spp. [16-22]. Sample sizes for rodent-based studies ranged from 10 to 502 . The prevalence ranged from $10 \%$ to $97 \%$, as seen in Table A1.

Studies identifying insects within the order Diptera as a source of Strongyloides spp. accounted for $2 \%$ of the published works, as seen in Table A1. Identified insects within this order included flies of the genus Musca spp. and Lucilia spp. All studies identifying Strongyloides spp. within Diptera identified it from sites within the continent of Africa [23-25]. The sample sizes ranged from 5000 to 9950, and prevalence was between $<1 \%$ and $2 \%$. 
Insects within the order Blattodea were identified in $2 \%$ of all studies, as seen in Table A1. Identified insects within this order include cockroaches from the genus Periplaneta spp. and Blattella spp. Four of the five identified studies reported Strongyloides spp. within populations of Blattodea in Africa. The remaining study identified Strongyloides spp. in Blattodea in Thailand. All studies identified infected insects within housing and food preparation areas [26-29]. The sample sizes of studies identifying insects within the order Blattodea ranged from 70 to 920 , with the prevalence ranging from $1 \%$ to $81 \%$.

Half $(50 \%)$ of published works identifying parasitic contamination of vegetables and fruits found S. stercoralis upon leafy, rough-surfaced vegetables such as lettuces, cabbage, celery, spinach, and carrot [30-37]. The sample sizes for fruit and vegetable-based studies ranged from 36 to 1130, with prevalence ranging from $<1 \%$ to $46 \%$.

Countries where Strongyloides spp. was identified in soils in public areas included Spain, Iran, Malaysia, Nigeria, Brazil, the Czech Republic, Slovakia, and Romania, as seen in Figure 2 [38-47]. Geophagy, the purposeful consumption of soils, was also commonly identified as a factor in infection from soil-based sources.

Studies identifying environmental sources of S. stercoralis, S. fuelleborni, and Strongyloides spp. are distributed across the world. Areas with a large amount of research included Europe, Africa, and South East Asia, as seen in Figure 1. Areas lacking research include Oceania, and the Americas, as seen in Figure 1. Many published studies identified Strongyloides spp. within temperate regions as opposed to tropical regions, as seen in Figure 1.

Microscopy was the most commonly used identification technique (90\%). However, molecular detection was more common in recent publications. For example, in 2018, 6 of the 14 papers identified employed molecular-based techniques; however, in 2011,1 of 11 papers published used molecular-based techniques, as seen in Table A1. 


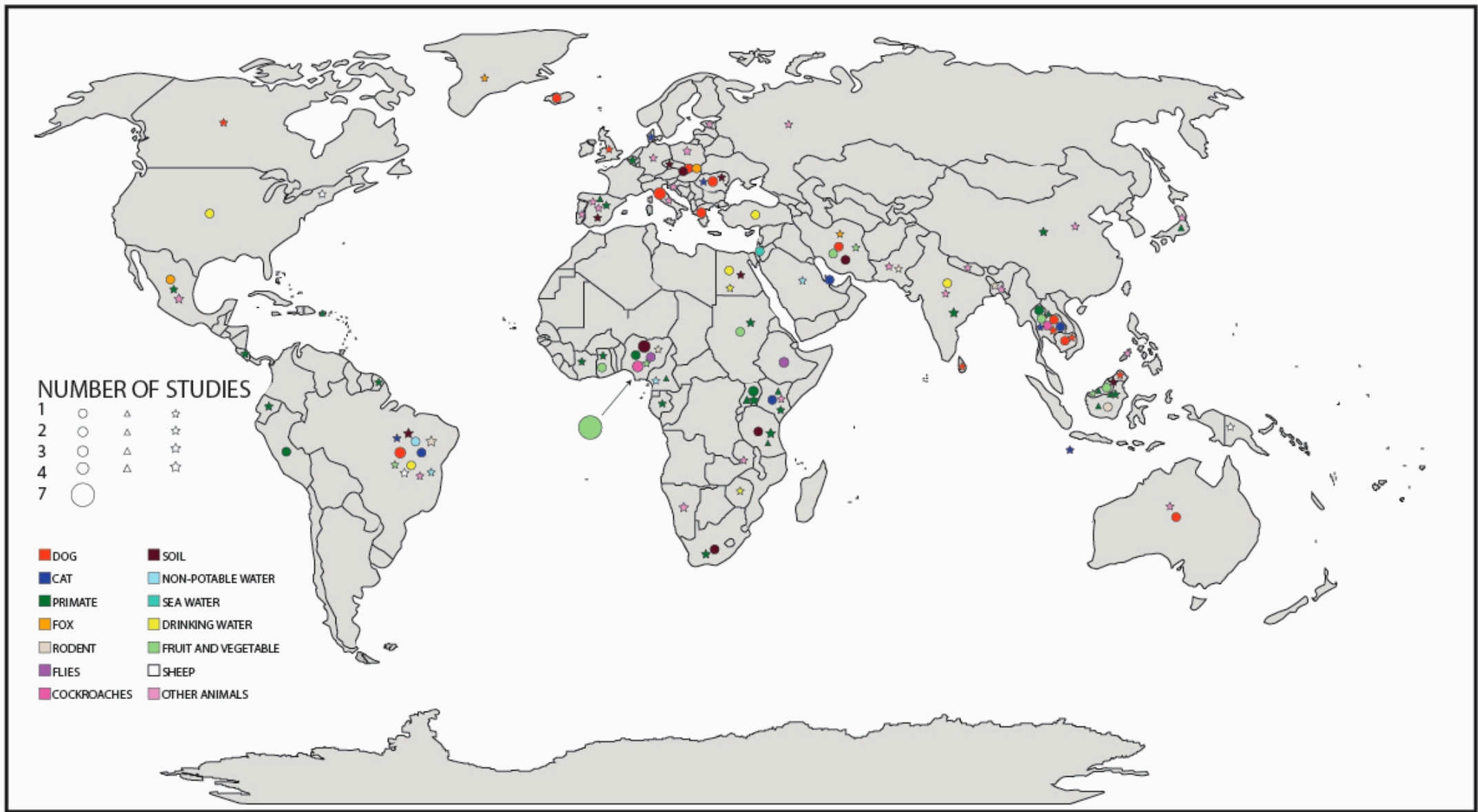

Figure 1. Map displaying the global distribution of all reported environmental cases of Strongyloides stercoralis, Strongyloides fuelleborni, and Strongyloides spp. Where circles are representative of Strongyloides stercoralis, diamonds are representative of Strongyloides fuelleborni, and stars are representative of Strongyloides spp. The size of each shape is mapped to the number of studies published in that country. Location of shapes does not represent exact location of study, but country in which the study was completed. Colored fill of shapes was assigned to a single source and is consistent across all helminth species. 


\section{Discussion}

\subsection{Animals}

Primates and domesticated or feral dogs (canids) adapt well to association with human settlements and cohabitation with humans, indicating the potential for transmission to humans. Contamination with feces from domesticated or synanthropic primates and dogs may lead to other environmental sources, such as water and soil, becoming reservoirs of Strongyloides spp. capable of causing infection. Most studies found in this review were based on primate and dog investigation, suggesting that these animals preferentially live closely with and benefit from humans. This habitual closeness presents a chance for environmental transmission of Strongyloides spp.

\subsubsection{Canids}

Studies that report parasites found in canid feces frequently investigate multiple parasites such as Ancylostoma spp., Giardia spp., and Strongyloides spp. These studies have often found low levels of S. stercoralis within otherwise highly parasitically infected populations [40,48-56]. Infection occurs more frequently in canids when they are living stray. This might be a result of exposure to infective Strongyloides spp. larvae occurring more frequently to these dogs than dogs living within homes [57]. Mass drug administration (MDA) to stray dogs has been implemented successfully for the control of rabies; accordingly, it may be an option for the control of Strongyloides spp. [10]. Isolated or infrequent anthelmintic treatment increases infection rates and so considered treatment must be implemented [10,58]. Studies identifying canid feces as containing Strongyloides spp. commonly also screened the samples for other parasites. Sample sizes ranged from 35 to 3465 . The highest prevalence was reported by Beknazarova et al. [59] who screened 35 canine fecal samples from Australia, of which $49 \%$ were positive for Strongyloides spp. This low sample size with a high positivity rate in comparison with other studies is representative of the inconsistent fecal shedding of Strongyloides spp. as well as the endemic location of the study. The lowest prevalence was reported by Ardelean et al. [60] with 1\% of 3465 samples positive from dogs within Romania. Strongyloidiasis was observed most commonly in dogs three to six months of age in this study. This variance based on age and study location may be further impacted by the detection method. Ardelean et al. [60] reported high levels of Ancylostomidae spp. which is morphologically similar to Strongyloides spp., therefore making reliable identification with microscopy alone difficult.

\subsubsection{Primates}

Areas sparsely populated by humans increase roaming in primates due to the attractive food sources but offer a low threat from the decreased human numbers. More frequent entry to communities in search of food potentially increases the numbers of Strongyloides spp.-infected primate feces within these sparsely populated communities $[13,14]$. Terrestrial Papio primates were likely to excrete Strongyloides spp. larvae; however, arboreal Cercopithecus neglectus were less likely [11,12]. This may be due to less frequent contact with soil containing Strongyloides spp. larvae. The impact of human populations upon forests has led to an increased chance of interaction between humans and potentially infected primates. Hasegawa et al. [61] observed that degraded forest increased the chance of roaming and transfer of parasites.

Captive primates present an infection risk to handlers because anthelmintic treatment has been observed to not eliminate Strongyloides spp. larvae shedding within feces [15]. This may be due to the introduction of new individuals to groups, a phenomenon also observed within wild individuals [15]. This indicates the value in introducing physical environmental controls beyond anthelmintic drugs, especially in communities exposed to roaming wild primates.

Tourist sanctuaries provide an ideal environment for contact between primates and humans. Environmental controls such as fecal contamination removal can decrease helminthic infection in both primates and humans without interfering with natural behaviors [62]. Strongyloides spp. is unable to 
transfer either from animal to human or from human to human directly [63]. This further supports the importance of clearing feces because contact with the animals does not cause infection; however, contact with fecal matter can cause infection. Larger groups, such as those within tourist sanctuaries, are generally associated with higher parasitic species richness. Some variation of infection can be expected based on food availability and stress levels [64].

Studies of primates had sample sizes ranging from 7 to 3349, with prevalence also ranging from $<1 \%$ to $100 \%$. Prevalence within primate populations was reported to be higher than in canine populations. Hasegawa et al. [61] reported $100 \%$ prevalence within seven gorilla and chimpanzees from Uganda; whereas Li et al. [65] screened 3349 fecal samples and identified a prevalence rate of 6\%. This variation in prevalence may be due to the inconsistent shedding of Strongyloides spp. larvae. Li et al. [65] employed microscopy whereas Hasegawa et al. [61] employed molecular techniques, which may account for differentiation in prevalence.

\subsubsection{Ruminants}

Strongyloides spp. has also been found within the feces of ruminants used in western farming settings including pigs, sheep, and cattle. All studies identifying Strongyloides spp. within farm-associated ruminants only identified the parasite to the species level $[66,67]$. These may have been genus specific, such as the pork threadworm, Strongyloides ransomi, or the more general Strongyloides papillosis. All studies used microscopy, a technique that can have low success in identifying Strongyloides spp. to species level. These recorded observations indicate the potential for infected ruminant feces to provide an environmental source of Strongyloides spp.

\subsubsection{Rodents}

Rodents are known to carry a range of communicable diseases. Strongyloides spp. has been found in several rodent species, including common house rats, and non-synanthropic rodents such as Hydrochoerus hydrochaeris. S. stercoralis has been identified in house rat feces in East Java, Indonesia, using microscopy [20]. The area in which S. stercoralis was identified in house rat feces is an area with poor sanitation and hygiene. People reported a large house rat population within these areas [20]. In such cases, where a zoonotic pathogen is identified, control of the offending carrier can be employed. The sample sizes of rodents were low in comparison with other sources, with the highest sample size being 502 [19]. The highest prevalence was within a population of Rattus norvegicus within a Brazilian slum [17]. The rodents sampled within this study had particularly high levels of infection with helminths; in all except five, helminths were present within their feces [17]. This prevalence of $97 \%$ from a sample size of 299 is representative of animals living within an area highly contaminated by human waste.

\subsubsection{Insects}

Increasing urbanization has allowed for synanthropic dependence to increase within insect populations. Densely urbanizing areas lead to an increase in available food for insects, and areas with poor sanitation and hygiene practices attract disease-carrying insects such as those within the order Blattodea (cockroaches) and Diptera (flies). Filth flies present a source of helminth transmission. Their preference for consuming wet, rotting substances indicates a high probability for the consumption and carriage of Strongyloides spp. Carriage of Strongyloides spp. has been observed on the external body of flies despite frequent preening and cleaning [68]. Fetene and Worku [23] identified S. stercoralis within Chrysomya rufifacies, Musca sorbens, and Lucilia cuprina. C. rufifacies were identified largely within butcheries and defecating grounds; $M$. sorbens was found more frequently within the market collection sites. Furthermore, Musca domestica, a species always found in association with humans, has been observed to carry Strongyloides spp. [24]. The presence of these flies within human food areas presents a potential transmission route for Strongyloides spp. larvae. Prevalence was higher in the internal structures of flies than on the external surface of flies [25]. This observation is further supported by 
the preference of these flies for consumption of wet substances. Insects within the order Blattodea, commonly known as cockroaches, also present a transmission source. Parasite prevalence has been found to be associated with housing type. Low-cost housing with pit latrines as well as housing in close proximity to dumpsites was reported to contain higher levels of carrier cockroaches [26,28,29]. Through the introduction of environmental controls such as fly screens or nets, movement of carrier insects can be decreased [27]. Sample sizes for both orders were high. Studies found low prevalence with the exception of Morenikeji et al. [28] who reported $81 \%$ in 70 cockroaches.

\subsection{Water}

Contamination of water is also a potential source of helminth transferal. Pollution of water sources with human and agricultural waste can render water sources unsuitable for use as drinking and irrigation water. In areas where water access is limited, contaminated water may be employed for these uses [69]. Waste stabilization ponds, chlorination, or activated sludge treatment systems may be suitable approaches for reducing helminth levels; however, many studies monitoring wastewater treatment methods have provided contradictory results [70-73]. Some studies identified standard treatment techniques as adequate for removing larvae; however, others did not. Frequent monitoring of treated waste water is important because treated water has been identified as containing higher than acceptable levels of helminths including Strongyloides spp. [74]. To date, studies have focused on the helminth burden of treated water instead of comparing treated with untreated levels. Through focusing on untreated and treated waters from the same area, reduction in burden levels of treated water may be better understood.

Untreated water used for drinking can contain Strongyloides spp., particularly when water is sourced from storm water or collected rainwater [75]. According to one study, when water runoff moves into drinking water sources such as rivers, it can carry Strongyloides spp. larvae with it [75]. Bore and ground-water contamination can also occur and has been identified [76,77]. Jonnalagadda and Bhat [77] found that improper washing of vessels used to collect and store water can lead to helminth contamination. Implementation of appropriate washing and sanitation education in areas with high contamination risk may decrease incidences of infection.

Prevalence of Strongyloides spp. within non-potable water was higher than in potable water; this was expected because most sources of non-potable water were collected from wastewater treatment facilities [71]. The prevalence of Strongyloides spp. within non-treated wastewater was between $40-100 \%$. Treated waste water intended for use on crops for human consumption had a much lower prevalence $(2 \%)$; however, it was still observed to be present, supporting the importance of monitoring water intended for reuse [70].

\subsection{Fruit and Vegetables}

The rough nature of green, leafy vegetables surfaces means that adhesion of parasitic larvae and eggs occurs easily when these vegetables are either washed with contaminated water or come into contact with contaminated human fecal-based fertilizers (i.e., night soil) [30]. Studies identified S. stercoralis contamination most frequently within leafy, rough-surfaced vegetables such as lettuces, cabbage, celery, spinach, and carrot [30-37]. This correlation may be due to these vegetables growing close to or in the ground, which may lead to increased contamination from fertilizers [78,79]. Market vendors commonly wash vegetables prior to purchase, and consumption of raw vegetables such as salad leaves is frequently noted $[31,79,80]$. There is an increasing focus on the study of vegetables, washing water, and farm soil to determine where in the food chain parasites are being introduced [33].

Prevalence of Strongyloides spp. within fruit and vegetable samples was generally low, ranging from $<1 \%$ to $46 \%$. Ogbolu et al. [81] found S. stercoralis in $46 \%$ of fresh vegetables sold at open markets in Nigeria. The application of night soils and untreated wastewater is common within low-income nations may have led to the high level of prevalence [81]. Lower prevalence was also reported within 
Nigeria, between $<1 \%$ and $19 \%$. This variation may be due to differences in handling of samples, treatment during farming, and cross contamination [30,31].

\subsection{Soil}

Increasing urbanization has led to an ever-increasing amount of waste. Modern waste includes not only fecal waste but waste produced in the form of rubbish. Dumpsites and landfills are commonly employed to deal with this large amount of waste; Strongyloides spp. contamination can occur throughout the nearby environments. Dumps and landfills pose a transmission risk due to the ability of Strongyloides spp. larvae to survive effectively in the soil [82].

Contamination of soils with animal feces within public recreation areas also presents a transmission source. High levels of soil-transmitted helminths were reported in public area soils such as parks in Spain, Iran, Malaysia, Nigeria, Brazil, the Czech Republic, Slovakia, and Romania [38-47].

The texture and chemistry of soil also plays a role in the prevalence of Strongyloides spp. larvae. Moisture levels in soils increases the incidence of rhabditiform larvae developing into filariform larvae [83]. This requirement for moisture is supported by the findings of a study of helminth larvae during the wet season, which found that no larvae were located during the dry season despite contamination [39]. High sand and silt content soils favor the survival of Strongyloides spp. and other helminth larvae. This is due to the high porosity of these soils, which allows larvae to move effectively through the soils towards sources of nutrition and moisture [84].

Strongyloides spp. is transmitted from soil-based sources to humans through skin-to-soil contact; however, the behavior of purposefully ingesting soil known as geophagy can also lead to soil-based infections. Geophagy is culturally accepted and common in sub-Saharan Africa. This behavior is common in pregnant women; S. stercoralis infections have been observed, along with other soil-transmitted helminth infections, in these women [85,86]. Geophagy may be undertaken as a method for diet supplementation in low-income areas. Notably, these areas are more likely to have helminth-contaminated soil, which leads to an increased chance of infection.

Prevalence of Strongyloides spp. within soil was between $1 \%$ and $20 \%$. Ivoke et al. [85] screened 797 pregnant women for parasitic infections related to geophagy. The prevalence of infection was $1 \%$ within this cohort. This low prevalence is likely due to picking soils specifically for consumption. Higher prevalence was observed in soils sampled in soil directly from areas densely populated with poor health infrastructure [41].

\subsection{PCR and Microscopy}

Identification of Strongyloides spp. nematodes can be undertaken using several methods. These fall into either techniques involving the identification of Strongyloides spp. larvae using a microscope or molecular-based techniques. Microscopy presents several problems but it accounts for most larval identification-based techniques $(90 \%)$ as presented in this literature review. This study did not exclude papers based on year; accordingly, this overrepresentation of microscopy is likely a result of the recent introduction of polymerase chain reaction (PCR) techniques. Recent papers have increasingly employed PCR as accessibility to the equipment increases. In 2018, 5 of the 14 papers published employed molecular-based techniques; in contrast, in 2011, only 1 of 11 studies published employed molecular-based techniques, as seen in Table A1. This increasing use of molecular-based techniques is expected, and its use will allow for more accurate identification of species of Strongyloides spp. The strengths of microscopy include that it can be employed within the field or where resources are limited; however, microscopy alone cannot reliably differentiate $S$. stercoralis from S. fuelleborni. The reliance on microscopy-based techniques is hazardous because both species are morphologically similar [34]. Molecular techniques allow for the accurate identification of Strongyloides spp. to the species level; however, set up of molecular protocols can be expensive. Each technique has strengths and weaknesses; when looking at all published works, a consideration of the identification techniques allows for more accurate assessment of reports. 


\subsection{Global Distribution}

Globally, published research has mainly focused in countries within Africa, Europe, and South East Asia. Commonly, Strongyloides spp. is reported as a tropical disease; however, Strongyloides spp. was often also reported in temperate regions such as Europe, as seen in Figure 1 [83,87]. This may be due to a lack of resources within low-income nations, leading to an overrepresentation of the generally higher income countries within Europe. Australia and the Americas both lacked studies looking at the environmental sources of Strongyloides spp. (Figure 1). This indicates a need for more research into the environmental transmission of S. stercoralis, S. fuelleborni, and Strongyloides spp. within these areas.

\section{Materials and Methods}

This systematic literature review is based on an adapted version of the PRISMA statement. This tool allows for the transparent and reliable reporting of evidence. A systematic search of the databases Scopus and Web of Science was undertaken, and all articles published prior to 2019 were included. Key words used in searches included Strongyloides spp., strongyloidiasis, tap water, soil, insect, zoonotic, and waste, as seen in Table 1. A search strategy was developed to ensure a transparent and complete literature review of all identified environmental sources of Strongyloides spp. was completed. This strategy is as follows; All non-English documents were excluded from the search.

To be included, published data must have reported Strongyloides spp. in one of the three spp. capable of human infection or to the genus level because these studies cannot be excluded as identifying disease-causing Strongyloides spp. The document must have reported this presence within an environmental source. Documents were excluded if they were reviews, reports of humans with Strongyloides spp. infection with no mention of contributing environmental source, or lab-based studies, as seen in Figure 2.

First, all titles and abstracts of all papers were manually reviewed to ensure the papers met inclusion criteria. If it was unclear from titles and abstracts if papers met the criteria, they were included for full text review. Papers that were unclear were included. Papers were then read as full text and compared against inclusion and exclusion criteria. Articles that met these criteria were included in the study. All papers included in the study had key points extracted and recorded including the environmental source reported, species of Strongyloides spp. observed, detection method used, and the country from which the sample was taken.

Table 1. Complete search strategy and all key words used to identify relevant literature.

\begin{tabular}{|c|}
\hline Search Terms Employed to Identify Relevant Literature \\
\hline $\begin{array}{l}\text { Strongyloides OR Strongyloidiasis OR "Strongyloides stercoralis" OR "S. stercoralis" OR "Strongyloides fuelleborni" } \\
\text { OR "S. fulleborni" OR "Strongyloides fulleborni kellyi" OR "S. fulleborni kellyi" }\end{array}$ \\
\hline $\begin{array}{l}\text { AND } \\
\text { "Tap Water" OR "Potable water" OR Water OR Soil OR Dirt OR sediment OR synanthropic OR "synanthropic } \\
\text { insect" OR Insect OR "Musca domestica" OR flies OR "Musca vetustissima" OR Sarcophagidae OR "Chrysomya } \\
\text { megacephala" OR "Musca sorbens" OR "Lucilia cuprina" OR "Calliphora vicina" OR "Blattella germanica" OR } \\
\text { "Periplaneta Americana" OR Cockroach OR dog OR "Canis lupis" OR zoonotic OR Monkey OR "septic tank" OR } \\
\text { waste OR wastewater OR rubbish OR trash OR environment }\end{array}$ \\
\hline
\end{tabular}



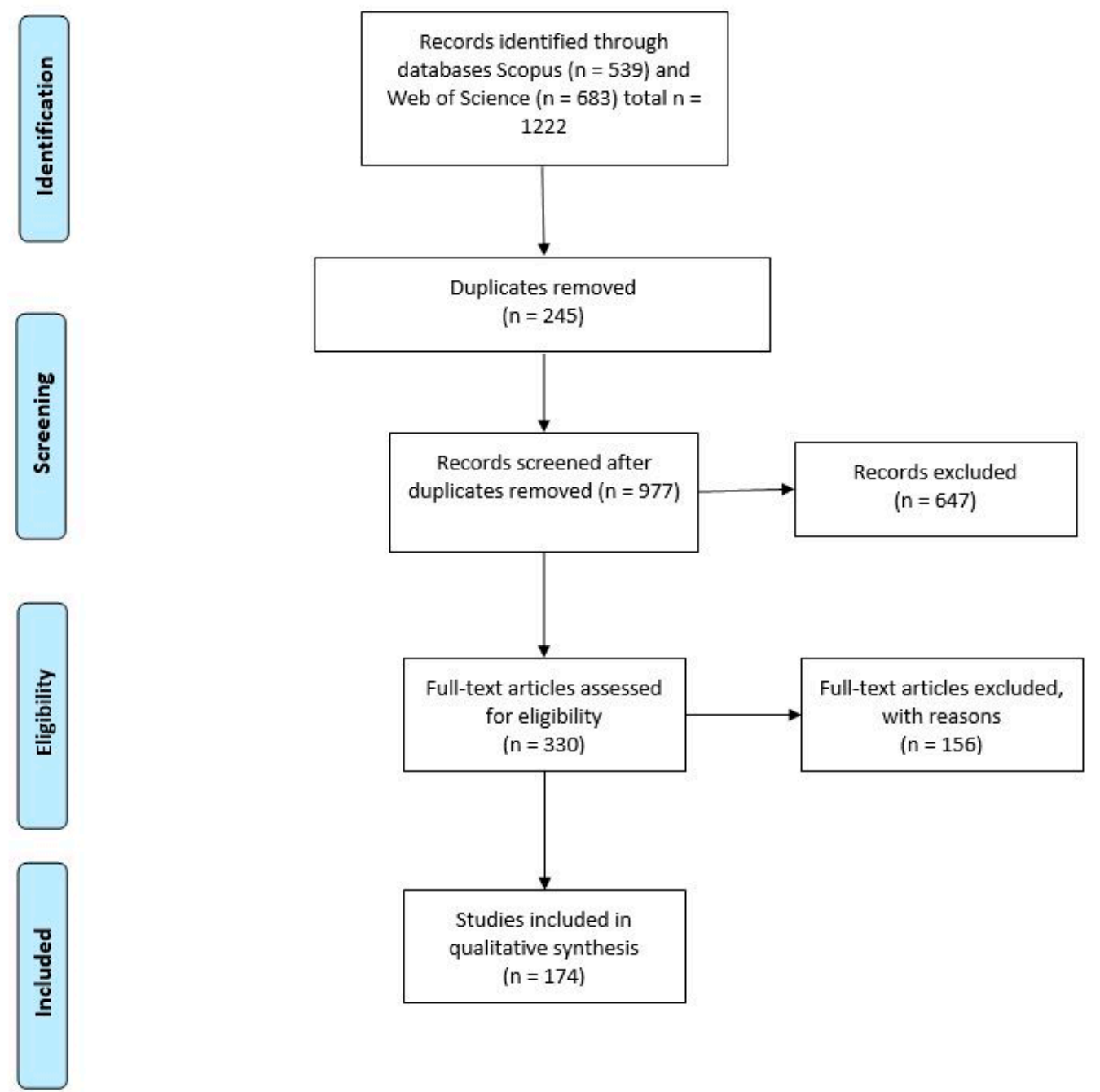

Studies included in $(n=174)$

Figure 2. Flow diagram representing the search strategies used (based on the PRISMA statement reporting guidelines for systematic literature reviews) showing an overview of the retrieved articles and the total articles identified as eligible.

\section{Conclusions}

Although Strongyloides spp. is considered a soil-transmitted helminth, there are several environmental sources that can potentially provide a route of transmission of the disease. Understanding the potential sources, combined with the adoption of environmental controls for Strongyloides spp. is likely to decrease transmission and therefore infections. Animals such as dogs, primates, and insects, as well as soil, water, and fruit and vegetables have all been reported to contain Strongyloides spp. larvae, capable of perpetuating infection within humans who have come into contact. Future research is needed to undertake a holistic screening of all environmental sources within endemic areas to identify those which pose the greatest significance to human health. By understanding the established and recorded environmental reservoirs of S. stercoralis, S. fuelleborni, and S. fuelleborni kellyi, better environmental controls can be implemented.

Author Contributions: M.A.F.W., H.W., and K.E.R. designed and participated in review design. M.A.F.W. drafted and edited the manuscript, H.W. corrected and contributed to the manuscript, K.E.R. corrected and contributed to the manuscript. All authors approved of the final manuscript.

Funding: This research received no external funding.

Acknowledgments: Thanks to Natasha White for producing the map using Adobe Illustrator.

Conflicts of Interest: The authors declare no conflict of interest. 


\section{Appendix A}

Table A1. Summary of all reports, and studies identifying S. stercoralis, S. fuelleborni, S. fuelleborni kellyi, and Strongyloides spp. within environmental sources worldwide.

\begin{tabular}{|c|c|c|c|c|c|c|}
\hline Species Parasite & Prevalence & Sample Size & Detection Method & Country & Reference & Source \\
\hline Strongyloides stercoralis & $1 \%$ & 3465 & Microscopy & Romania & (Ardelean et al., 2005) [60] & Dog \\
\hline Strongyloides stercoralis & $49 \%$ & 35 & Molecular & Australia & (Beknazarova et al., 2017) [59] & Dog \\
\hline Strongyloides stercoralis & $<1 \%$ & 3208 & Microscopy & Iceland & (Eydal and Skirnisson 2016) [88] & Dog \\
\hline Strongyloides stercoralis & $<1 \%$ & 215 & Microscopy & Brazil & (Ferreira et al., 2006) [89] & Dog \\
\hline Strongyloides spp. & $<1 \%$ & 457 & Microscopy & Canada & (Gaunt and Carr 2011) [90] & Dog \\
\hline Strongyloides stercoralis & $<1 \%$ & 181 & Microscopy & Brazil & (Goncalves et al., 2007) [91] & Dog \\
\hline Strongyloides stercoralis & $87 \%$ & 88 & Molecular & Cambodia & (Jaleta et al., 2017) [6] & Dog \\
\hline Strongyloides stercoralis & $<1 \%$ & 879 & Microscopy & Greece & (Kostopoulou et al., 2017) [48] & Dog \\
\hline Strongyloides stercoralis & $<1 \%$ & 189 & Microscopy & Thailand & (Leelayoova et al., 2009) [49] & Dog \\
\hline Strongyloides stercoralis & $45 \%$ & 171 & Microscopy & Brazil & (Martins et al., 2012) [58] & Dog \\
\hline Strongyloides stercoralis & $4 \%$ & 52 & Microscopy & Romania & (Mircean et al., 2012) [92] & Dog \\
\hline Strongyloides spp. & $5 \%$ & 175 & Microscopy & Malaysia & (Noor Azian et al., 2008) [40] & Dog \\
\hline Strongyloides stercoralis & $2 \%$ & 281 & Microscopy & Greece & (Papazahariadouet al., 2007) [50] & Dog \\
\hline Strongyloides stercoralis & $2 \%$ & 272 & Microscopy & Italy & (Paradies et al., 2017) [93] & Dog \\
\hline Strongyloides spp. & $11 \%$ & 90 & Microscopy & Sri Lanka & (Perera et al., 2013) [57] & Dog \\
\hline Strongyloides stercoralis & $6 \%$ & 174 & Microscopy & Iran & (Razmi et al., 2009) [52] & Dog \\
\hline Strongyloides stercoralis & $<1 \%$ & 239 & Microscopy & Italy & (Riggio et al., 2013) [53] & Dog \\
\hline Strongyloides stercoralis & $<1 \%$ & 639 & Microscopy & Italy & (Sauda et al., 2018) [55] & Dog \\
\hline Strongyloides spp. & $15 \%$ & 94 & Microscopy & Cambodia & (Schär et al.,2014) [87] & Dog \\
\hline Strongyloides stercoralis & $10 \%$ & 60 & Microscopy & Slovakia & (Štrkolcová et al., 2017) [45] & Dog \\
\hline Strongyloides spp. & $2 \%$ & 171 & Microscopy & England & (Wright et al., 2016) [56] & Dog \\
\hline Strongyloides stercoralis & $<1 \%$ & 463 & Microscopy & Italy & (Zanzani et al., 2014) [94] & Dog \\
\hline Strongyloides spp. & $2 \%$ & 197 & Microscopy & Thailand & (Pumidonming et al., 2016) [51] & Dog \\
\hline Strongyloides stercoralis & $18 \%$ & 824 & Microscopy & Qatar & (Abu-Madi et al., 2007) [95] & Cat \\
\hline Strongyloides spp. & $47 \%$ & 28 & Microscopy & Christmas Island & (Adams et al., 2008) [96] & Cat \\
\hline Strongyloides spp. & $54 \%$ & 37 & Microscopy & Brazil & (Lima et al., 2017) [97] & Cat \\
\hline Strongyloides spp. & $3 \%$ & 414 & Microscopy & Romania & (Mircean et al., 2010) [98] & Cat \\
\hline Strongyloides stercoralis & $14 \%$ & 173 & Microscopy & Brazil & (Monteiro et al., 2016) [99] & Cat \\
\hline Strongyloides stercoralis & $44 \%$ & 103 & Microscopy & Kenya & (Njuguna et al., 2017) [100] & Cat \\
\hline Strongyloides spp. & $<1 \%$ & 300 & Microscopy & Thailand & (Rojekittikhun et al., 2014) [101] & Cat \\
\hline
\end{tabular}


Table A1. Cont.

\begin{tabular}{|c|c|c|c|c|c|c|}
\hline Species Parasite & Prevalence & Sample Size & Detection Method & Country & Reference & Source \\
\hline Strongyloides stercoralis & $21 \%$ & 38 & Microscopy & Thailand & $\begin{array}{l}\text { (Sedionoto and Anamnart 2018) } \\
\text { [102] }\end{array}$ & Cat \\
\hline Strongyloides spp. & $991.0 \%$ & 99 & Microscopy & Denmark & (Takeuchi-Storm et al., 2015) [103] & Cat \\
\hline Strongyloides fuelleborni & UNK & UNK & $\begin{array}{l}\text { Molecular and } \\
\text { Microscopy }\end{array}$ & Japan & (Arizono et al., 2012) [13] & Primate \\
\hline Strongyloides spp. & $4144 \%$ & 41 & Microscopy & Uganda & (Bezjian et al., 2008) [11] & Primate \\
\hline Strongyloides spp. & $37 \%$ & 24 & Microscopy & French Guiana & (De Thoisy et al., 2001) [14] & Primate \\
\hline Strongyloides spp. & $21 \%$ & 125 & Microscopy & India & (Ekanayake et al., 2006) [104] & Primate \\
\hline Strongyloides fuelleborni & $28 \%$ & 293 & Microscopy & Uganda & (Gillespie et al., 2004) [105] & Primate \\
\hline $\begin{array}{c}\text { Strongyloides fuelleborni } \\
\text { and Strongyloides } \\
\text { stercoralis }\end{array}$ & $\begin{array}{l}<1 \% \text { S. } \\
\text { stercoralis, } 4 \% \\
\text { S. fuelleborni }\end{array}$ & 2103 & Microscopy & Uganda & (Gillespie et al., 2005) [106] & Primate \\
\hline Strongyloides fuelleborni & $84 \%$ & 153 & Microscopy & Tanzania & (Gillespie et al., 2010) [107] & Primate \\
\hline $\begin{array}{l}\text { Strongyloides fuelleborni } \\
\text { and Strongyloides spp. }\end{array}$ & $\begin{array}{l}11 \% \text { S. } \\
\text { fulleborni, } 15 \% \\
\text { S. spp }\end{array}$ & 27 & Microscopy & Spain & (Gomez et al., 1996) [15] & Primate \\
\hline Strongyloides fuelleborni & $23 \%$ & 401 & Microscopy & Japan & (Gotoh 2000) [108] & Primate \\
\hline $\begin{array}{c}\text { Strongyloides fuelleborni } \\
\text { and Strongyloides } \\
\text { stercoralis }\end{array}$ & $100 \%$ & 7 & Molecular & Uganda & (Hasegawa et al., 2016) [61] & Primate \\
\hline Strongyloides spp. & $88 \%$ & 96 & Microscopy & Ecuador & (Helenbrook et al., 2015) [64] & Primate \\
\hline Strongyloides spp. & $4 \%$ & 238 & Microscopy & Uganda & (Hodder and Chapman 2012) [109] & Primate \\
\hline Strongyloides spp. & $7 \%$ & 40 & Microscopy & Kenya & (Karere and Munene 2002) [12] & Primate \\
\hline Strongyloides spp. & $41 \%$ & 624 & Microscopy & Borneo & (Klaus et al., 2018) [62] & Primate \\
\hline Strongyloides fuelleborni & $32 \%$ & 652 & Microscopy & Borneo & (Klaus et al., 2017) [110] & Primate \\
\hline Strongyloides fuelleborni & $57 \%$ & 141 & Microscopy & Puerto Rico & (Knezevich et al., 1998) [111] & Primate \\
\hline Strongyloides spp. & $43 \%$ & 686 & Microscopy & Tanzania & (Kooriyama et al., 2012) [112] & Primate \\
\hline Strongyloides spp. & $74 \%$ & 3142 & Microscopy & Côte d'Ivoire & (Kouassi et al., 2015 [113] & Primate \\
\hline Strongyloides spp. & $13 \%$ & 366 & Microscopy & India & (Kumar et al., 2018) [114] & Primate \\
\hline Strongyloides fuelleborni & $\begin{array}{l}44 \% \text { S. } \\
\text { fuelleborni, } 4 \% \\
\text { S. spp. }\end{array}$ & 25 & Microscopy & Malaysia & (Kuze et al., 2010) [115] & Primate \\
\hline Strongyloides fuelleborni & $95 \%$ & 20 & $\begin{array}{l}\text { Molecular and } \\
\text { Microscopy }\end{array}$ & Indonesia & (Labes et al., 2011) [116] & Primate \\
\hline
\end{tabular}


Table A1. Cont.

\begin{tabular}{|c|c|c|c|c|c|c|}
\hline Species Parasite & Prevalence & Sample Size & Detection Method & Country & Reference & Source \\
\hline Strongyloides spp. & $37 \%$ & 59 & Microscopy & Ethiopia & (Legesse and Erko 2004) [117] & Primate \\
\hline Strongyloides spp. & $5 \%$ & 222 & Microscopy & Belgium & (Levecke et al., 2007) [118] & Primate \\
\hline Strongyloides spp. & $6 \%$ & 3349 & microscopy & China & (Li et al., 2017) [65] & Primate \\
\hline Strongyloides stercoralis & $6 \%$ & 46 & Microscopy & Nigeria & (Mafuyai et al., 2013) [119] & Primate \\
\hline Strongyloides spp. & $50 \%$ & 134 & Microscopy & Costa Rica & (Maldonado-Lopez et al., 2014) [120] & Primate \\
\hline Strongyloides spp. & $77 \%$ & 78 & Microscopy & Ecuador & (Martin-Solano et al., 2017) [121] & Primate \\
\hline Strongyloides spp. & $17 \%$ & 53 & Microscopy & Uganda & (Matsubayashi et al., 1992) [122] & Primate \\
\hline Strongyloides fuelleborni & $58 \%$ & 432 & Molecular & Uganda & (McLennan et al., 2017) [123] & Primate \\
\hline Strongyloides spp. & $84 \%$ & 121 & Microscopy & Uganda & (Muehlenbein et al., 2005) [124] & Primate \\
\hline Strongyloides fuelleborni & $45 \%$ & 315 & Microscopy & Kenya & (Munene et al., 1998) [125] & Primate \\
\hline Strongyloides fuelleborni & $21 \%$ & 297 & Microscopy & Kenya & (Muriuki et al., 1998) [126] & Primate \\
\hline Strongyloides spp. & $76 \%$ & 83 & Microscopy & Costa Rica & (Parr et al., 2013) [127] & Primate \\
\hline Strongyloides spp. & $13 \%$ & 366 & Microscopy & Tanzania & (Petrasova et al., 2010) [128] & Primate \\
\hline Strongyloides spp. & $44 \%$ & 130 & Microscopy & Tanzania & (Petrzelkova et al., 2010) [129] & Primate \\
\hline Strongyloides stercoralis & $15 \%$ & 86 & Microscopy & Peru & (Phillips et al., 2004) [130] & Primate \\
\hline Strongyloides spp. & $43 \%$ & 47 & Microscopy & Gabon & (Pouillevet et al., 2017) [131] & Primate \\
\hline Strongyloides fuelleborni & $6 \%$ & 125 & Microscopy & Cameroon & (Pourrut et al., 2011) [132] & Primate \\
\hline Strongyloides spp. & $53 \%$ & 55 & Microscopy & Ghana & (Ryan et al., 2012) [133] & Primate \\
\hline Strongyloides spp. & $8 \%$ & 420 & Molecular & Mexico & $\begin{array}{c}\text { (Solorzano-Garcia and de Leon 2017) } \\
\text { [134] }\end{array}$ & Primate \\
\hline Strongyloides fuelleborni & $39 \%$ & 243 & Molecular & Thailand and Laos & (Thanchomnang et al., 2018) [135] & Primate \\
\hline Strongyloides spp. & $35 \%$ & 283 & Microscopy & India & (Tiwari et al., 2017) [136] & Primate \\
\hline Strongyloides stercoralis & $31 \%$ & 135 & Microscopy & Thailand & (Wenz-Mucke et al., 2013) [137] & Primate \\
\hline Strongyloides spp. & $24 \%$ & 272 & Microscopy & South Africa & (Wren et al., 2015) [138] & Primate \\
\hline Strongyloides spp. & $24 \%$ & 332 & Microscopy & South Africa & (Wren et al., 2016) [139] & Primate \\
\hline $\begin{array}{l}\text { Strongyloides fuelleborni } \\
\text { and Strongyloides spp. }\end{array}$ & UNK & 14 & Molecular & Malaysian Borneo & (Frias et al., 2018) [140] & Primate \\
\hline Strongyloides spp. & $29 \%$ & 64 & Microscopy & Brazil & (De Souza et al., 2012) [66] & Sheep \\
\hline Strongyloides spp. & $8 \%$ & 165 & Microscopy & $\begin{array}{l}\text { Papua New } \\
\text { Guinea }\end{array}$ & (Koinari et al., 2013) [141] & Sheep \\
\hline Strongyloides spp. & $<1 \%$ & 27 & Microscopy & New England & (MacGlaflin et al., 2011) [142] & Sheep \\
\hline Strongyloides spp. & UNK & 1798 & Microscopy & Brazil & (McManus et al., 2009) [143] & Sheep \\
\hline Strongyloides spp. & $2 \%$ & 275 & Microscopy & Greenland & (Andreassen et al., 2017) [144] & Fox \\
\hline Strongyloides spp. & $4 \%$ & 22 & Microscopy & Iran & (Dalimi et al., 2006) [145] & Fox \\
\hline
\end{tabular}


Table A1. Cont.

\begin{tabular}{|c|c|c|c|c|c|c|}
\hline Species Parasite & Prevalence & Sample Size & Detection Method & Country & Reference & Source \\
\hline Strongyloides stercoralis & $16 \%$ & 249 & Microscopy & Mexico & $\begin{array}{c}\text { (Hernandez-Camacho et al., 2011) } \\
\text { [146] }\end{array}$ & Fox \\
\hline Strongyloides stercoralis & $2 \%$ & 1198 & Microscopy & Slovakia & (Miterpakova et al., 2009) [147] & Fox \\
\hline Strongyloides spp. & $65 \%$ & 60 & Microscopy & Pakistan & (Afshan et al., 2013) [16] & Rat \\
\hline Strongyloides spp. & $97 \%$ & 299 & Microscopy & Brazil & (Carvalho-Pereira et al., 2018) [17] & Rat \\
\hline Strongyloides spp. & $40 \%$ & 25 & Microscopy & Brazil & (Lima et al., 2017) [97] & Rat \\
\hline Strongyloides spp. & $13 \%$ & 76 & Microscopy & Bangladesh & (Fuehrer et al., 2012) [18] & Rat \\
\hline Strongyloides spp. & $10 \%$ & 502 & Microscopy & Nigeria & (Isaac et al., 2018) [19] & $\begin{array}{l}\text { Mouse } \\
\text { and rat }\end{array}$ \\
\hline Strongyloides stercoralis & $53 \%$ & 98 & Microscopy & Indonesia & (Prasetyo et al., 2016) [20] & House rat \\
\hline Strongyloides spp. & $10 \%$ & 10 & Microscopy & Brazil & (Souza et al., 2015) [21] & Capybaras \\
\hline Strongyloides spp. & $10 \%$ & 31 & Microscopy & Brazil & (Gioia-Di Chiacchio et al., 2014) [22] & Capybaras \\
\hline Strongyloides stercoralis & $2 \%$ & 6530 & Microscopy & Ethiopia & (Fetene and Worku 2009) [23] & Flies \\
\hline Strongyloides stercoralis & $<1 \%$ & 9950 & Microscopy & Ethiopia & (Getachew et al., 2007) [24] & Flies \\
\hline Strongyloides stercoralis & $2 \%$ & 5000 & Microscopy & Nigeria & (Umeche 1989b) [25] & Flies \\
\hline Strongyloides stercoralis & $12 \%$ & 749 & Microscopy & Nigeria & (Adenusi et al., 2018) [26] & Cockroaches \\
\hline Strongyloides stercoralis & $1 \%$ & 920 & Microscopy & Thailand & (Chamavit et al., 2010) [27] & Cockroaches \\
\hline Strongyloides stercoralis & $81 \%$ & 70 & Microscopy & Nigeria & (Morenikeji et al., 2016) [28] & Cockroaches \\
\hline Strongyloides stercoralis & UNK & 234 & Microscopy & Nigeria & (Tatfeng et al., 2005) [29] & Cockroaches \\
\hline Strongyloides stercoralis & $2 \%$ & 125 & Microscopy & Nigeria & (Adesewa and Morenikeji, 2017) [82] & Soil \\
\hline Strongyloides spp. & $3 \%$ & 625 & Microscopy & Spain & (Dado et al., 2012) [38] & Soil \\
\hline Strongyloides spp. & $8 \%$ & 120 & Microscopy & Egypt & (Etewa et al., 2016) [83] & Soil \\
\hline Strongyloides stercoralis & $1 \%$ & 797 & Microscopy & Nigeria & (Ivoke et al., 2017) [85] & Geophagy \\
\hline Strongyloides stercoralis & $2 \%$ & 1078 & Microscopy & Tanzania & (Kawai et al., 2009) [86] & Geophagy \\
\hline Strongyloides stercoralis & $3 \%$ & 112 & Microscopy & Iran & (Motazedian et al., 2006) [39] & Soil \\
\hline Strongyloides spp. & $7 \%$ & 182 & Microscopy & Malaysia & (Noor Azian et al., 2008) [40] & Soil \\
\hline Strongyloides stercoralis & $20 \%$ & 102 & Microscopy & Nigeria & (Ogbolu et al., 2011) [41] & Soil \\
\hline Strongyloides spp. & $5 \%$ & 2520 & Microscopy & Brazil & (Rocha et al., 2011) [42] & Soil \\
\hline Strongyloides spp. & $2 \%$ & 500 & Microscopy & Czech Republic & (Valkounova 1982) [43] & Soil \\
\hline Strongyloides spp. & $3 \%$ & 125 & Microscopy & Brazil & (Mandarino-Pereira et al., 2010) [44] & Soil \\
\hline Strongyloides stercoralis & $14 \%$ & 14 & Microscopy & Slovakia & (Strkolcova et al., 2017) [45] & Soil \\
\hline
\end{tabular}


Table A1. Cont.

\begin{tabular}{|c|c|c|c|c|c|c|}
\hline Species Parasite & Prevalence & Sample Size & Detection Method & Country & Reference & Source \\
\hline Strongyloides stercoralis & $12 \%$ & 17 & Microscopy & South Africa & (Sumbele et al., 2014) [84] & Soil \\
\hline Strongyloides spp. & $4 \%$ & 45 & Microscopy & Romania & (Tudor 2015) [46] & Soil \\
\hline Strongyloides stercoralis & $6 \%$ & 150 & Microscopy & Nigeria & (Umeche 1989a) [47] & Soil \\
\hline Strongyloides spp. & $6 \%$ & 16 & Microscopy & Brazil & (da Silva et al., 2014) [148] & Soil \\
\hline Strongyloides spp. & UNK & 8 & Microscopy & Cameroon & (Aghaindum and Landry, 2019) [149] & $\begin{array}{c}\text { Non-potable } \\
\text { water }\end{array}$ \\
\hline Strongyloides spp. & $40 \%-100 \%$ & 100 & Microscopy & Saudi Arabia & (Bolbol 1992) [69] & $\begin{array}{c}\text { Non-potable } \\
\text { water }\end{array}$ \\
\hline Strongyloides stercoralis & $2 \% \%$ & UNK & Microscopy & Brazil & (Bastos et al., 2008) [70] & $\begin{array}{c}\text { Non-potable } \\
\text { water }\end{array}$ \\
\hline Strongyloides spp. & $100 \%$ & 3 & Microscopy & Brazil & (Cutolo et al., 2006) [71] & $\begin{array}{c}\text { Non-potable } \\
\text { water }\end{array}$ \\
\hline Strongyloides stercoralis & $19 \%$ & 52 & Microscopy & Palestine & (Hilles et al., 2014) [150] & Seawater \\
\hline Strongyloides stercoralis & $1 \%$ & 85 & Microscopy & Turkey & (Bakir et al., 2003) [151] & $\begin{array}{c}\text { Drinking } \\
\text { water }\end{array}$ \\
\hline $\begin{array}{l}\text { Strongyloides fuelleborni } \\
\text { and Strongyloides spp. }\end{array}$ & $\begin{array}{c}11 \% \text { S. } \\
\text { fuelleborni, } 15 \% \\
\text { S. spp. }\end{array}$ & 9950 & Microscopy & Zimbabwe & (Dalu et al., 2011) [76] & $\begin{array}{l}\text { Drinking } \\
\text { water }\end{array}$ \\
\hline Strongyloides spp. & UNK & UNK & Microscopy & Egypt & (El Shazly et al., 2003) [75] & $\begin{array}{l}\text { Drinking } \\
\text { water }\end{array}$ \\
\hline Strongyloides stercoralis & $7 \%$ & 80 & Microscopy & Egypt & (El-Badry et al., 2018) [152] & $\begin{array}{c}\text { Drinking } \\
\text { water }\end{array}$ \\
\hline Strongyloides stercoralis & $81 \%$ & 16 & Microscopy & Brazil & (Freitas et al., 2017) [153] & $\begin{array}{l}\text { Drinking } \\
\text { water }\end{array}$ \\
\hline Strongyloides stercoralis & $51 \%$ & 232 & Microscopy & India & (Jonnalagadda and Bhat 1995) [77] & $\begin{array}{l}\text { Drinking } \\
\text { water }\end{array}$ \\
\hline Strongyloides stercoralis & $100 \%$ & UNK & Microscopy & USA & (Klotz et al., 1992) [154] & $\begin{array}{l}\text { Drinking } \\
\text { water }\end{array}$ \\
\hline Strongyloides stercoralis & UNK & UNK & Molecular & Malaysia & (Zeehaida et al., 2011) [155] & $\begin{array}{c}\text { Fruit \& } \\
\text { vegetables }\end{array}$ \\
\hline Strongyloides stercoralis & $<1 \%$ & 1130 & Microscopy & Nigeria & (Adamu et al., 2012) [30] & $\begin{array}{c}\text { Fruit \& } \\
\text { vegetables }\end{array}$ \\
\hline
\end{tabular}


Table A1. Cont.

\begin{tabular}{|c|c|c|c|c|c|c|}
\hline Species Parasite & Prevalence & Sample Size & Detection Method & Country & Reference & Source \\
\hline Strongyloides stercoralis & $<1 \%$ & 960 & Microscopy & Nigeria & (Adenusi et al., 2015) [31] & $\begin{array}{c}\text { Fruit } \& \\
\text { vegetables }\end{array}$ \\
\hline Strongyloides stercoralis & $10 \%$ & 150 & Microscopy & Nigeria & (Amaechi et al., 2016) [78] & $\begin{array}{c}\text { Fruit \& } \\
\text { vegetables }\end{array}$ \\
\hline Strongyloides stercoralis & $7 \%$ & 190 & Microscopy & Nigeria & (Amuta et al., 2017) [32] & $\begin{array}{c}\text { Fruit \& } \\
\text { vegetables }\end{array}$ \\
\hline Strongyloides stercoralis & $7 \%$ & 240 & Microscopy & Nigeria & (Dada et al., 2015) [33] & $\begin{array}{c}\text { Fruit } \& \\
\text { vegetables }\end{array}$ \\
\hline Strongyloides spp. & $1 \%$ & 453 & Microscopy & Iran & (Fallah et al., 2016) [34] & $\begin{array}{c}\text { Fruit \& } \\
\text { vegetables }\end{array}$ \\
\hline Strongyloides stercoralis & $36 \%$ & 360 & Microscopy & Ghana & (Kudah et al., 2018) [35] & $\begin{array}{c}\text { Fruit \& } \\
\text { vegetables }\end{array}$ \\
\hline Strongyloides spp. & $13 \%$ & 108 & Microscopy & Brazil & (Luz et al., 2017) [156] & $\begin{array}{c}\text { Fruit \& } \\
\text { vegetables }\end{array}$ \\
\hline Strongyloides spp. & $19 \%$ & 199 & Microscopy & Nigeria & (Maikai et al., 2012) [157] & $\begin{array}{c}\text { Fruit \& } \\
\text { vegetables }\end{array}$ \\
\hline Strongyloides spp. & $11 \%$ & 36 & Microscopy & Malaysia & (Matyusof et al., 2017) [80] & $\begin{array}{c}\text { Fruit \& } \\
\text { vegetables }\end{array}$ \\
\hline Strongyloides stercoralis & $1 \%$ & 260 & Microscopy & Sudan & (Mohamed et al., 2016) [36] & $\begin{array}{c}\text { Fruit \& } \\
\text { vegetables }\end{array}$ \\
\hline Strongyloides stercoralis & $10 \%$ & 265 & Microscopy & Thailand & (Punsawad et al., 2019) [37] & $\begin{array}{c}\text { Fruit \& } \\
\text { vegetables }\end{array}$ \\
\hline Strongyloides stercoralis & $46 \%$ & 120 & Microscopy & Nigeria & (Ogbolu et al., 2009) [81] & $\begin{array}{c}\text { Fruit \& } \\
\text { vegetables }\end{array}$ \\
\hline Strongyloides stercoralis & $14 \%$ & 140 & Microscopy & Iran & (Madadi 2010) [158] & $\begin{array}{c}\text { Fruit \& } \\
\text { vegetables }\end{array}$ \\
\hline Strongyloides stercoralis & $19 \%$ & 80 & Microscopy & Nigeria & (Ohaeri and Unogu 2011) [79] & $\begin{array}{c}\text { Fruit \& } \\
\text { vegetables }\end{array}$ \\
\hline Strongyloides spp. & $7 \%$ & 15 & Microscopy & Zambia & (Berentsen et al., 2012) [159] & $\begin{array}{l}\text { Other } \\
\text { animals }\end{array}$ \\
\hline Strongyloides spp. & $5 \%$ & 272 & Microscopy & Nepal & (Bista et al., 2017) [160] & $\begin{array}{l}\text { Other } \\
\text { animals }\end{array}$ \\
\hline
\end{tabular}


Table A1. Cont.

\begin{tabular}{|c|c|c|c|c|c|c|}
\hline Species Parasite & Prevalence & Sample Size & Detection Method & Country & Reference & Source \\
\hline Strongyloides spp. & $100 \%$ & 1 & Microscopy & Brazil & (Cardia et al., 2016) [161] & $\begin{array}{c}\text { Other } \\
\text { animals }\end{array}$ \\
\hline Strongyloides spp. & $4 \%$ & 432 & Microscopy & Spain & (Cordon et al., 2008) [162] & $\begin{array}{c}\text { Other } \\
\text { animals }\end{array}$ \\
\hline Strongyloides spp. & $40 \%$ & 52 & Microscopy & Russia & (González et al., 2007) [163] & $\begin{array}{l}\text { Other } \\
\text { animals }\end{array}$ \\
\hline Strongyloides spp. & $2 \%$ & 956 & Microscopy & India & (Gupta et al., 2018) [164] & $\begin{array}{c}\text { Other } \\
\text { animals }\end{array}$ \\
\hline Strongyloides spp. & $<1 \%$ & 1005 & Microscopy & Germany & (Hallinger et al., 2018) [165] & $\begin{array}{c}\text { Other } \\
\text { animals }\end{array}$ \\
\hline Strongyloides spp. & $31 \%$ & 42 & Microscopy & Japan & (Hasegawa et al., 2017) [166] & $\begin{array}{c}\text { Other } \\
\text { animals }\end{array}$ \\
\hline Strongyloides spp. & $<1 \%$ & 400 & Microscopy & Croatia & (Hermosilla et al., 2017) [167] & $\begin{array}{c}\text { Other } \\
\text { animals }\end{array}$ \\
\hline Strongyloides spp. & $64 \%-99 \%$ & 990 & Microscopy & Mexico & (Hu et al., 2018) [168] & $\begin{array}{c}\text { Other } \\
\text { animals }\end{array}$ \\
\hline Strongyloides spp. & $4 \%$ & 821 & Microscopy & China & (Huang et al., 2014) [169] & $\begin{array}{c}\text { Other } \\
\text { animals }\end{array}$ \\
\hline Strongyloides spp. & $15 \%$ & 2280 & Microscopy & Pakistan & (Khan et al., 2010) [67] & $\begin{array}{c}\text { Other } \\
\text { animals }\end{array}$ \\
\hline Strongyloides spp. & UNK & 6 & Microscopy & Namibia & (Kumba et al., 2003) [170] & $\begin{array}{c}\text { Other } \\
\text { animals }\end{array}$ \\
\hline Strongyloides spp. & $36 \%$ & 58 & Microscopy & Poland & (Mizgajska-Wiktor et al., 2010) [171] & $\begin{array}{c}\text { Other } \\
\text { animals }\end{array}$ \\
\hline Strongyloides spp. & $67 \%$ & 12 & Microscopy & Mexico & (Mukul-Yerves et al., 2014) [172] & $\begin{array}{c}\text { Other } \\
\text { animals }\end{array}$ \\
\hline Strongyloides spp. & $57 \%$ & 201 & Microscopy & Estonia & (Oja et al., 2017) [173] & $\begin{array}{c}\text { Other } \\
\text { animals }\end{array}$ \\
\hline Strongyloides spp. & $47 \%$ & 383 & Microscopy & Mexico & (Ojeda-Robertos et al., 2017) [174] & $\begin{array}{c}\text { Other } \\
\text { animals }\end{array}$ \\
\hline Strongyloides spp. & $7 \%$ & 6 & Molecular & Iberian Peninsula & (Perera et al., 2013) [175] & $\begin{array}{c}\text { Other } \\
\text { animals }\end{array}$ \\
\hline
\end{tabular}


Table A1. Cont.

\begin{tabular}{|c|c|c|c|c|c|c|}
\hline Species Parasite & Prevalence & Sample Size & Detection Method & Country & Reference & Source \\
\hline Strongyloides spp. & $3 \%$ & 468 & Microscopy & Poland & (Pilarczyk et al., 2015) [176] & $\begin{array}{c}\text { Other } \\
\text { animals }\end{array}$ \\
\hline Strongyloides spp. & $17 \%$ & 86 & Microscopy & Bangladesh & (Rahman et al., 2018) [177] & $\begin{array}{c}\text { Other } \\
\text { animals }\end{array}$ \\
\hline Strongyloides spp. & $3 \%$ & 1883 & Microscopy & Italy & (Rinaldi et al., 2009) [178] & $\begin{array}{c}\text { Other } \\
\text { animals }\end{array}$ \\
\hline Strongyloides spp. & $44 \%$ & 163 & Microscopy & Portugal & (Rosalino et al., 2006) [179] & $\begin{array}{c}\text { Other } \\
\text { animals }\end{array}$ \\
\hline Strongyloides spp. & $45 \%$ & 82 & Microscopy & Australia & (Turni and Smales 2001) [180] & $\begin{array}{l}\text { Other } \\
\text { animals }\end{array}$ \\
\hline Strongyloides spp. & UNK & UNK & Microscopy & Namibia & (Turner et al., 2010) [181] & $\begin{array}{l}\text { Other } \\
\text { animals }\end{array}$ \\
\hline Strongyloides spp. & UNK & UNK & Microscopy & Namibia & (Turner et al., 2012) [182] & $\begin{array}{c}\text { Other } \\
\text { animals }\end{array}$ \\
\hline Strongyloides spp. & $<1 \%$ & 213 & Microscopy & Kenya & (VanderWaal et al., 2014) [183] & $\begin{array}{c}\text { Other } \\
\text { animals }\end{array}$ \\
\hline Strongyloides spp. & $74 \%$ & 243 & Microscopy & Philippines & (Ybanez et al., 2018) [184] & $\begin{array}{c}\text { Other } \\
\text { animals }\end{array}$ \\
\hline
\end{tabular}




\section{References}

1. Olsen, A.; Van Lieshout, L.; Marti, H.; Polderman, T.; Polman, K.; Steinmann, P.; Stothard, R.; Thybo, S.; Verweij, J.J.; Magnussen, P. Strongyloidiasis—The most neglected of the neglected tropical diseases? Trans. R. Soc. Trop. Med. Hyg. 2009, 103, 967-972. [CrossRef] [PubMed]

2. Ashford, R.W.; Barnish, G.; Viney, M.E. Strongyloides fuelleborni kellyi: Infection and disease in Papua New Guinea. Parasitol. Today 1992, 8, 314-318. [CrossRef]

3. Hauber, H.P.; Galle, J.; Chiodini, P.L.; Rupp, J.; Birke, R.; Vollmer, E.; Zabel, P.; Lange, C. Fatal outcome of a hyperinfection syndrome despite successful eradication of Strongyloides with subcutaneous ivermectin. Infect. 2005, 33, 383-386. [CrossRef]

4. Kearns, T.M.; Currie, B.J.; Cheng, A.C.; McCarthy, J.; Carapetis, J.R.; Holt, D.C.; Page, W.; Shield, J.; Gundjirryirr, R.; Mulholland, E.; et al. Strongyloides seroprevalence before and after an ivermectin mass drug administration in a remote Australian Aboriginal community. PLoS Negl. Trop. Dis. 2017, 11, e0005607. [CrossRef] [PubMed]

5. Vadlamudi, R.S.; Chi, D.S.; Krishnaswamy, G. Intestinal strongyloidiasis and hyperinfection syndrome. Clin. Mol. Allergy 2006, 4, 8. [CrossRef] [PubMed]

6. Jaleta, T.G.; Zhou, S.; Bemm, F.M.; Schär, F.; Khieu, V.; Muth, S.; Odermatt, P.; Lok, J.B.; Streit, A. Different but overlapping populations of Strongyloides stercoralis in dogs and humans-Dogs as a possible source for zoonotic strongyloidiasis. PLoS Negl. Trop. Dis. 2017, 11. [CrossRef] [PubMed]

7. Bisoffi, Z.; Buonfrate, D.; Montresor, A.; Requena-Méndez, A.; Muñoz, J.; Krolewiecki, A.J.; Gotuzzo, E.; Mena, M.A.; Chiodini, P.L.; Anselmi, M.; et al. Strongyloides stercoralis: A plea for action. PLoS Negl. Trop. Dis. 2013, 7, e2214. [CrossRef]

8. Jourdan, P.M.; Lamberton, P.H.L.; Fenwick, A.; Addiss, D.G. Soil-transmitted helminth infections. Lancet 2018, 391, 252-265. [CrossRef]

9. Maroto, R.; Jiménez, A.E.; Romero, J.J.; Alvarez, V.; De Oliveira, J.B.; Hernández, J. First report of anthelmintic resistance in gastrointestinal nematodes of sheep from Costa Rica. Vet. Med. Int. 2011, 2011, 145312. [CrossRef]

10. Beknazarova, M.; Whiley, H.; Ross, K. Mass drug administration for the prevention human strongyloidiasis should consider concomitant treatment of dogs. PLoS Negl. Trop. Dis. 2017, 11, e0005735. [CrossRef]

11. Bezjian, M.; Gillespie, T.R.; Chapman, C.A.; Greiner, E.C. Coprologic evidence of gastrointestinal helminths of forst baboons, Papio anubis, in Kibale national park, Uganda. J. Wildl. Dis. 2008, 44, 878-887. [CrossRef] [PubMed]

12. Karere, G.M.; Munene, E. Some gastro-intestinal tract parasites in wild De Brazza's monkeys (Cercopithecus neglectus) in Kenya. Vet. Parasitol. 2002, 110, 153-157. [CrossRef]

13. Arizono, N.; Yamada, M.; Tegoshi, T.; Onishi, K. Molecular identification of oesophagostomum and trichuris eggs isolated from wild Japanese macaques. Korean J. Parasitol. 2012, 50, 253-257. [CrossRef] [PubMed]

14. De Thoisy, B.; Vogel, I.; Reynes, J.M.; Pouliquen, J.F.; Carme, B.; Kazanji, M.; Vié, J.C. Health evaluation of translocated free-ranging primates in French Guiana. Am. J. Primatol. 2001, 54, 1-16. [CrossRef] [PubMed]

15. Gomez, M.S.; Gracenea, M.; Montoliu, I.; Feliu, C.; Monleon, A.; Fernandez, J.; Ensenat, C. Intestinal parasitism-Protozoa and helminths-In primates at the Barcelona zoo. J. Med. Primatol. 1996, 25, 419-423. [CrossRef]

16. Afshan, K.; Beg, M.A.; Rizvi, S.S.R.; Qayyum, M. Helminths and nematode infection in Norway rats (Rattus norvegicus) captured from Northern Punjab, Pakistan. Pak. J. Zool. 2013, 45, 1456-1459.

17. Carvalho-Pereira, T.; Souza, F.N.; Santos, L.R.N.; Walker, R.; Pertile, A.C.; de Oliveira, D.S.; Pedra, G.G.; Minter, A.; Rodrigues, M.G.; Bahiense, T.C.; et al. The helminth community of a population of Rattus norvegicus from an urban Brazilian slum and the threat of zoonotic diseases. Parasitol. 2018, 145, 797-806. [CrossRef]

18. Fuehrer, H.P.; Baumann, T.A.; Riedl, J.; Treiber, M.; Igel, P.; Swoboda, P.; Joachim, A.; Noedl, H. Endoparasites of rodents from the Chittagong hill tracts in Southeastern Bangladesh. Wien. Klin. Wochenschr. 2012, 124, 27-30. [CrossRef]

19. Isaac, C.; Igbinosa, B.I.; Ohiolei, J.A.; Osimen, C.E. Endoparasites of small mammals in Edo State, Nigeria: Public health implications. Korean J. Parasitol. 2018, 56, 93-100. [CrossRef] 
20. Prasetyo, R.H. Survey of house rat intestinal parasites from Surabaya District, East Java, Indonesia that can cause opportunistic infections in humans. Southeast Asian J. Trop. Med. Public Health 2016, 47, 194-198.

21. Souza, G.T.R.; Ribeiro, T.S.; Antonucci, A.M.; Ueda, B.H.; Carniel, M.K.; Karling, L.C.; Eiras, J.C.; Takemoto, R.M.; Pavanelli, G.C. Endoparasite fauna of wild capybaras (Hydrochoerus hydrochaeris) (linnaeus, 1766) from the Upper Parana River floodplain, Brazil. Aquat. Mamm. 2015, 41, 213-221. [CrossRef]

22. Gioia-Di Chiacchio, R.; Prioste, F.E.S.; Vanstreels, R.E.T.; Knobl, T.; Kolber, M.; Miyashiro, S.I.; Matushima, E.R. Health evaluation and survey of zoonotic pathogens in free-ranging capybaras (Hydrochoerus hydrochaeris). J. Wildl. Dis. 2014, 50, 496-504. [CrossRef] [PubMed]

23. Fetene, T.; Worku, N. Public health importance of non-biting cyclorrhaphan flies. Trans. R. Soc. Trop. Med. Hyg. 2009, 103, 187-191. [CrossRef] [PubMed]

24. Getachew, S.; Gebre-Michael, T.; Erko, B.; Balkew, M.; Medhin, G. Non-biting cyclorrhaphan flies (diptera) as carriers of intestinal human parasites in slum areas of Addis Ababa, Ethiopia. Acta Trop. 2007, 103, 186-194. [CrossRef] [PubMed]

25. Umeche, N.; Mandah, L.E. Musca domestica as a carrier of intestinal helminths in Calabar, Nigeria. East. Afr. Med. J. 1989, 66, 349-352.

26. Adenusi, A.A.; Akinyemi, M.I.; Akinsanya, D. Domiciliary cockroaches as carriers of human intestinal parasites in Lagos metropolis, Southwest Nigeria: Implications for public health. J. Arthropod-Borne Dis. 2018, 12, 141-151. [CrossRef] [PubMed]

27. Chamavit, P.; Sahaisook, P.; Niamnuy, N. The majority of cockroaches from the Samutprakarn province of Thailand are carriers of parasitic organisms. EXCLI J. 2011, 10, 218-222.

28. Morenikeji, O.A.; Adebiyi, A.; Oluwayiose, O.A. Parasites in cockroaches recovered from residential houses around awotan dumpsite in Ido local government area of Oyo State, Nigeria. Annu. Res. Rev. Biol. 2016, 9. [CrossRef]

29. Tatfeng, Y.M.; Usuanlele, M.U.; Orukpe, A.; Digban, A.K.; Okodua, M.; Oviasogie, F.; Turay, A.A. Mechanical transmission of pathogenic organisms: The role of cockroaches. J. Vector Borne Dis. 2005, 42, 129-134.

30. Adamu, N.B.; Adamu, J.Y.; Mohammed, D. Prevalence of helminth parasites found on vegetables sold in Maiduguri, Northeastern Nigeria. Food Control 2012, 25, 23-26. [CrossRef]

31. Adenusi, A.A.; Abimbola, W.A.; Adewoga, T.O.S. Human intestinal helminth contamination in pre-washed, fresh vegetables for sale in major markets in Ogun State, Southwest Nigeria. Food Control 2015, 50, 843-849. [CrossRef]

32. Amuta, E.U.; Obisike, V.U.; Acham, N.I. Prevalence of helminth eggs on raw vegetables and fruits sold in selected markets in Makurdi, Benue State, Nigeria. Annu. Res. Rev. Biol. 2017, 19. [CrossRef]

33. Dada, A.J.; Wartu, J.R.; Auta, T.; Diya, A.W. Public health significance of helminthes eggs isolated from raw vegetables obtained from farms and those sold within Kaduna Metropolis, Nigeria. Asian J. Microbiol. Biotechnol. Environ. Sci. 2015, 17, 527-532.

34. Fallah, A.A.; Makhtumi, Y.; Pirali-Kheirabadi, K. Seasonal study of parasitic contamination in fresh salad vegetables marketed in Shahrekord, Iran. Food Control 2016, 60, 538-542. [CrossRef]

35. Kudah, C.; Sovoe, S.; Baiden, F. Parasitic contamination of commonly consumed vegetables in two markets in Ghana. Ghana Med. J. 2018, 52, 88-93. [CrossRef]

36. Mohamed, M.A.; Siddig, E.E.; Elaagip, A.H.; Edris, A.M.M.; Nasr, A.A. Parasitic contamination of fresh vegetables sold at central markets in Khartoum State, Sudan. Ann. Clin. Microb. Antimicrob. 2016, 15. [CrossRef] [PubMed]

37. Punsawad, C.; Phasuk, N.; Thongtup, K.; Nagavirochana, S.; Viriyavejakul, P. Prevalence of parasitic contamination of raw vegetables in Nakhon Si Thammarat province, Southern Thailand. BMC Public Health 2019, 19. [CrossRef]

38. Dado, D.; Izquierdo, F.; Vera, O.; Montoya, A.; Mateo, M.; Fenoy, S.; Galvan, A.L.; Garcia, S.; Garcia, A.; Aranguez, E.; et al. Detection of zoonotic intestinal parasites in public parks of Spain. Potential Epidemiological Role of Microsporidia. Zoonoese Public Health 2012, 59, 23-28. [CrossRef]

39. Motazedian, H.; Mehrabani, D.; Tabatabaee, S.H.R.; Pakniat, A.; Tavalali, M. Prevalence of helminth ova in soil samples from public places in Shiraz. East. Med. Health J. 2006, 12, 562-565.

40. Noor Azian, M.Y.; Sakhone, L.; Hakim, S.L.; Yusri, M.Y.; Nurulsyamzawaty, Y.; Zuhaizam, A.H.; Rodi, I.M.; Maslawaty, M.N. Detection of helminth infections in dogs and soil contamination in rural and urban areas. Southeast Asian J. Trop. Med. Public Health 2008, 39, 205-212. 
41. Ogbolu, D.O.; Alli, O.A.; Amoo, A.O.; Olaosun, I.I.; Ilozavbie, G.W.; Olusoga-Ogbolu, F.F. High-level parasitic contamination of soil sampled in Ibadan Metropolis. Afr. J. Med. Med. Sci. 2011, 40, 321-325. [PubMed]

42. Rocha, S.; Pinto, R.M.F.; Floriano, A.P.; Teixeira, L.H.; Bassili, B.; Martinez, A.; da Costa, S.O.P.; Caseiro, M.M. Environmental analyses of the parasitic profile found in the sandy soil from the Santos municipality beaches, sp, Brazil. Rev. Inst. Med. Trop. Sao Paulo 2011, 53, 277-281. [CrossRef] [PubMed]

43. Valkounová, J. Parasitological investigation of children's sandboxes and dog faeces from public areas of housing development in Prague. Folia Parasitol. 1982, 29, 133-138. [PubMed]

44. Mandarino-Pereira, A.; de Souza, F.S.; Lopes, C.W.G.; Pereira, M.J.S. Prevalence of parasites in soil and dog feces according to diagnostic tests. Vet. Parasitol. 2010, 170, 176-181. [CrossRef] [PubMed]

45. Štrkolcová, G.; Goldová, M.; Bocková, E.; Mojžišová, J. The roundworm Strongyloides stercoralis in children, dogs, and soil inside and outside a segregated settlement in Eastern Slovakia: Frequent but hardly detectable parasite. Parasitol. Res. 2017, 116, 891-900. [CrossRef]

46. Tudor, P. Soil Contamination with canine intestinal parasites eggs in the parks and shelter dogs from Bucharest Area. Agric. Agric. Sci. Procedia 2015, 6, 387-391. [CrossRef]

47. Umeche, N. Helminth ova in soil from children's playgrounds in Calabar, Nigeria. Central Afr. J. Med. 1989, 35, 432-434.

48. Kostopoulou, D.; Claerebout, E.; Arvanitis, D.; Ligda, P.; Voutzourakis, N.; Casaert, S.; Sotiraki, S. Abundance, zoonotic potential and risk factors of intestinal parasitism amongst dog and cat populations: The scenario of Crete, Greece. Parasit. Vectors 2017, 10. [CrossRef]

49. Leelayoova, S.; Siripattanapipong, S.; Naaglor, T.; Taamasri, P.; Mungthin, M. Prevalence of intestinal parasitic infections in military personnel and military dogs, Thailand. J. Med. Assoc. Thail. Chotmaihet Thangphaet 2009, 92 (Suppl. 1), S53-S59.

50. Papazahariadou, A.; Founta, A.; Papadopoulos, E.; Chliounakis, S.; Antoniadou-Sotiriadou, K.; Theodorides, Y. Gastrointestinal parasites of shepherd and hunting dogs in the Serres Prefecture, Northern Greece. Vet. Parasitol. 2007, 148, 170-173. [CrossRef]

51. Pumidonming, W.; Salman, D.; Gronsang, D.; Abdelbaset, A.E.; Sangkaeo, K.; Kawazu, S.; Igarashi, M. Prevalence of gastrointestinal helminth parasites of zoonotic significance in dogs and cats in lower Northern Thailand. J.Vet. Med. Sci. 2016, 78, 1779-1784. [CrossRef] [PubMed]

52. Razmi, G.R. Survey of dogs' parasites in Khorasan Razavi province, Iran. Iran. J. Parasitol. 2009, 4, 48-54.

53. Riggio, F.; Mannella, R.; Ariti, G.; Perrucci, S. Intestinal and lung parasites in owned dogs and cats from central Italy. Vet. Parasitol. 2013, 193, 78-84. [CrossRef] [PubMed]

54. Santos, J.L.C.; Magalhanes, N.B.; dos Santos, H.A.; Ribeiro, R.R.; Guimaraes, M.P. Parasites of domestic and wild canids in the region of Serra Do Cipo national park, Brazil. Rev. Bras. De Parasitol. Vet. 2012, 21, 270-277. [CrossRef]

55. Sauda, F.; Malandrucco, L.; Macri, G.; Scarpulla, M.; De Liberato, C.; Terracciano, G.; Fichi, G.; Berrilli, F.; Perrucci, S. Leishmania infantum, dirofilaria spp. And other endoparasite infections in kennel dogs in central Italy. Parasite 2018, 25. [CrossRef]

56. Wright, I.; Stafford, K.; Coles, G. The prevalence of intestinal nematodes in cats and dogs from Lancashire, North-West England. J. Small Anim. Pract. 2016, 57, 393-395. [CrossRef]

57. Perera, P.K.; Rajapakse, R.; Rajakaruna, R.S. Gastrointestinal parasites of dogs in Hantana area in the Kandy district. J. National Sci. Found. Sri Lanka 2013, 41, 81-91. [CrossRef]

58. Martins, C.M.; de Barros, C.D.; Bier, D.; Marinho, A.P.; Figueiredo, J.M.G.; Hoffmann, J.L.; Molento, M.B.; Biondo, A.W. Dog parasite incidence and risk factors, from sampling after one-year interval, in Pinhais, Brazil. Rev. Bras. De Parasitol. Vet. 2012, 21, 101-106. [CrossRef]

59. Beknazarova, M.; Millsteed, S.; Robertson, G.; Whiley, H.; Ross, K. Validation of dess as a DNA preservation method for the detection of Strongyloides spp. In canine feces. Int. J. Environmen. Res. Public Health 2017, 14, 624. [CrossRef]

60. Ardelean, A.I.; Suteu, E.; Cozma, V. Epidemiology of digestive helminthosis from urban area of Clujnapoca, Romania. Bull. Univ. Agric. Sci. Vet. Med. Vet. Med. 2005, 62, 322-329.

61. Hasegawa, H.; Kalousova, B.; McLennan, M.R.; Modry, D.; Profousova-Psenkova, I.; Shutt-Phillips, K.A.; Todd, A.; Huffman, M.A.; Petrzelkova, K.J. Strongyloides infections of humans and great apes in Dzangasangha protected areas, Central African republic and in degraded forest fragments in Bulindi, Uganda. Parasitol. Int. 2016, 65, 367-370. [CrossRef] [PubMed] 
62. Klaus, A.; Strube, C.; Roper, K.M.; Radespiel, U.; Schaarschmidt, F.; Nathan, S.; Goossens, B.; Zimmermann, E. Fecal parasite risk in the endangered proboscis monkey is higher in an anthropogenically managed forest environment compared to a Riparian rain forest in Sabah, Borneo. PLoS ONE 2018, 13. [CrossRef] [PubMed]

63. Grove, D.I. Strongyloidiasis: Is it transmitted from husband to wife? Br. J. Vener. Dis. 1982, 58, $271-272$. [CrossRef] [PubMed]

64. Helenbrook, W.D.; Wade, S.E.; Shields, W.M.; Stehman, S.V.; Whipps, C.M. Gastrointestinal parasites of Ecuadorian mantled howler monkeys (Alouatta palliata aequatorialis) based on fecal analysis. J. Parasitol. 2015, 101, 341-350. [CrossRef] [PubMed]

65. Li, J.Q.; Dong, H.J.; Wang, R.J.; Yu, F.C.; Wu, Y.Y.; Chang, Y.K.; Wang, C.R.; Qi, M.; Zhang, L.X. An investigation of parasitic infections and review of molecular characterization of the intestinal protozoa in nonhuman primates in China from 2009 to 2015. Int. J. Parasitol. Parasit. Wildl. 2017, 6, 8-15. [CrossRef] [PubMed]

66. de Souza, M.D.; Pimentel-Neto, M.; da Silva, R.M.; Farias, A.C.B.; Guimaraes, M.P. Gastrointestinal parasites of sheep, municipality of Lajes, Rio Grande Do Norte, Brazil. Rev. Bras. De Parasitol. Vet. 2012, 21, 71-73. [CrossRef]

67. Khan, M.N.; Sajid, M.S.; Khan, M.K.; Iqbal, Z.; Hussain, A. Gastrointestinal helminthiasis: Prevalence and associated determinants in domestic ruminants of district Toba Tek Singh, Punjab, Pakistan. Parasitol. Res. 2010, 107, 787-794. [CrossRef]

68. Förster, M.; Klimpel, S.; Sievert, K. The house fly (Musca domestica) as a potential vector of metazoan parasites caught in a pig-pen in Germany. Vet. Parasitol. 2009, 160, 163-167. [CrossRef]

69. Bolbol, A.S. Risk of contamination of human and agricultural environment with parasites through reuse of treated municipal wastewater in Riyadh, Saudi Arabia. J. Hyg. Epidemiol. Microbiol. Immunol. 1992, 36, 330-337.

70. Bastos, R.K.X.; Bevilacqua, P.D.; Silva, C.A.B.; Silva, C.V. Wastewater irrigation of salad crops: Further evidence for the evaluation of the WHO guidelines. Water Sci. Technol. 2008, 57, 1213-1219. [CrossRef]

71. Cutolo, S.A.; Matté, M.H.; Rocha, A.A. Monitoring of parasitological contamination in treated wastewater from activated sludge system. Manag. Environ. Qual. Int. J. 2006, 17, 43-56. [CrossRef]

72. Saqer, A.S.; Seham, H.; Ragaa, G.; Yehia, A.E.W.; Wafaa, S. Optimum methods of inactivation of Strongyloides stercoralis larvae from reclaimed wastewater. Environ. Monit. Assess. 2007, 130, 341-346. [CrossRef] [PubMed]

73. Tonani, K.A.A.; Julião, F.C.; Trevilato, T.M.B.; Takayanagui, A.M.M.; Bocio, A.; Domingo, J.L.; Segura-Muñoz, S.I. Behavior of metals, pathogen parasites, and indicator bacteria in sewage effluents during biological treatment by activated sludge. Biol. Trace Elem. Res. 2011, 143, 1193-1201. [CrossRef] [PubMed]

74. Hatam-Nahavandi, K.; Mahvi, A.H.; Mohebali, M.; Keshavarz, H.; Mobedi, I.; Rezaeian, M. Detection of parasitic particles in domestic and urban wastewaters and assessment of removal efficiency of treatment plants in Tehran, Iran. J. Environ. Health Sci. Eng. 2015, 13. [CrossRef] [PubMed]

75. El Shazly, A.M.; Soliman, M.; Nemr, H.I.; El Moafyo, N.; Abel Gawad, A.G.; El Bendary, M. Nematodes and water pollution in eastern part of Nile Delta. J. Egy. Soc. Parasitol. 2003, 33, 631-636.

76. Dalu, T.; Barson, M.; Nhiwatiwa, T. Impact of intestinal microorganisms and protozoan parasites on drinking water quality in Harare, Zimbabwe. J. Water Sanit. Hyg. Dev. 2011, 1, 153-163. [CrossRef]

77. Jonnalagadda, P.R.; Bhat, R.V. Parasitic contamination of stored water used for drinking/cooking in Hyderabad. Southeast Asian J. Trop. Med. Public Health 1995, 26, 789-794.

78. Amaechi, E.C.; Ohaeri, C.C.; Ukpai, O.M.; Adegbite, R.A. Prevalence of parasitic contamination of salad vegetables in Ilorin, North Central, Nigeria. Momona Ethiop. J. Sci. 2016, 8, 136-145. [CrossRef]

79. Ohaeri, C.C.; Unogu, L.O. Soil transmitted helminths of some common fruits and vegetables in Umuahia, Abia State Nigeria. Niger. J. Parasitol. 2011, 32, 305-308.

80. Matyusof, A.; Mohammad, M.; Abshir Abdullahi, M.; Mohamed, Z.; Zakaria, R.; Abdul Wahab, R. Occurrence of intestinal parasitic contamination in select consumed local raw vegetables and fruits in Kuantan, Pahang. Trop. Life Sci. Res. 2017, 28, 23-32. [CrossRef]

81. Ogbolu, D.O.; Alli, O.A.; Ogunleye, V.F.; Olusoga-Ogbolu, F.F.; Olaosun, I. The presence of intestinal parasites in selected vegetables from open markets in South Western Nigeria. Afr. J. Med. Med. Sci. 2009, 38, 319-324. [PubMed] 
82. Adesewa, A.; Morenikeji, O. Helminths and heavy metals in soils from a dumpsite in Ibadan City, Nigeria. J. Prev. Med. Hyg. 2017, 58, E328-E333. [PubMed]

83. Etewa, S.E.; Abdel-Rahman, S.A.; Abd El-Aal, N.F.; Fathy, G.M.; El-Shafey, M.A.; Ewis, A.M.G. Geohelminths distribution as affected by soil properties, physicochemical factors and climate in Sharkyia Governorate Egypt. J. Parasit. Dis. 2016, 40, 496-504. [CrossRef] [PubMed]

84. Sumbele, I.U.; Ngole, V.M.; Ekosse, G.I.E. Influence of physico-chemistry and mineralogy on the occurrence of geohelminths in geophagic soils from selected communities in the Eastern cape, South Africa, and their possible implication on human health. Int. J. Environ. Health Res. 2014, 24, 18-30. [CrossRef] [PubMed]

85. Ivoke, N.; Ikpor, N.; Ivoke, O.; Ekeh, F.; Ezenwaji, N.; Odo, G.; Iyaji, F.; Onoja, U.; Eyo, J. Geophagy as risk behaviour for gastrointestinal nematode infections among pregnant women attending antenatal clinics in a humid tropical zone of Nigeria. Afr. Health Sci. 2017, 17, 24-31. [CrossRef] [PubMed]

86. Kawai, K.; Saathoff, E.; Antelman, G.; Msamanga, G.; Fawzi, W.W. Geophagy (soil-eating) in relation to anemia and helminth infection among HIV-infected pregnant women in Tanzania. Am. J. Trop. Med. Hyg. 2009, 80, 36-43. [CrossRef]

87. Schär, F.; Trostdorf, U.; Giardina, F.; Khieu, V.; Muth, S.; Marti, H.; Vounatsou, P.; Odermatt, P. Strongyloides stercoralis: Global distribution and risk factors. PLoS Negl. Trop. Dis. 2013, 7, e2288. [CrossRef]

88. Eydal, M.; Skirnisson, K. Strongyloides stercoralis found in imported dogs, household dogs and kennel dogs in Iceland. Icel. Agric. Sci. 2016, 29, 39-51. [CrossRef]

89. Ferreira, A.; Goncalves-Pires, M.R.F.; Silva, D.A.O.; Goncalves, A.L.R.; Costa-Cruz, J.M. Parasitological and serological diagnosis of Strongyloides stercoralis in domesticated dogs from Southeastern Brazil. Vet. Parasitol. 2006, 136, 137-145. [CrossRef]

90. Gaunt, M.C.; Carr, A.P. A survey of intestinal parasites in dogs from Saskatoon, Saskatchewan. Can. Vet. J. Revue Vet. Can. 2011, 52, 497-500.

91. Goncalves, A.L.R.; Machado, G.A.; Goncalves-Pires, M.R.F.; Ferreira-Junior, A.; Silva, D.A.O.; Costa-Cruz, J.M. Evaluation of strongyloidiasis in kennel dogs and keepers by parasitological and serological assays. Vet. Parasitol. 2007, 147, 132-139. [CrossRef] [PubMed]

92. Mircean, V.; Gyorke, A.; Cozma, V. Prevalence and risk factors of Giardia duodenalis in dogs from Romania. Vet. Parasitol. 2012, 184, 325-329. [CrossRef] [PubMed]

93. Paradies, P.; Iarussi, F.; Sasanelli, M.; Capogna, A.; Lia, R.P.; Zucca, D.; Greco, B.; Cantacessi, C.; Otranto, D. Occurrence of strongyloidiasis in privately owned and sheltered dogs: Clinical presentation and treatment outcome. Parasit. Vectors 2017, 10. [CrossRef] [PubMed]

94. Zanzani, S.A.; Di Cerbo, A.R.; Gazzonis, A.L.; Genchi, M.; Rinaldi, L.; Musella, V.; Cringoli, G.; Manfredi, M.T. Canine fecal contamination in a metropolitan area (Milan, North-Western Italy): Prevalence of intestinal parasites and evaluation of health risks. Sci. World J. 2014, 2014. [CrossRef] [PubMed]

95. Abu-Madi, M.A.; Al-Ahbabi, D.A.; Al-Mashhadani, M.M.; Al-Ibrahim, R.; Pal, P.; Lewis, J.W. Patterns of parasitic infections in faecal samples from stray cat populations in Qatar. J. Helminthol. 2007, 81, 281-286. [CrossRef]

96. Adams, P.J.; Elliot, A.D.; Algar, D.; Brazell, R.I. Gastrointestinal parasites of feral cats from Christmas Island. Aust. Vet. J. 2008, 86, 60-63. [CrossRef] [PubMed]

97. Lima, V.F.S.; Ramos, R.A.N.; Lepold, R.; Borges, J.C.G.; Ferreira, C.D.; Rinaldi, L.; Cringoli, G.; Alves, L.C. Gastrointestinal parasites in feral cats and rodents from the Fernando De Noronha Archipelago, Brazil. Rev. Bras. Parasit. Vet. 2017, 26, 521-524. [CrossRef]

98. Mircean, V.; Titilincu, A.; Vasile, C. Prevalence of endoparasites in household cat (felis catus) populations from Transylvania (Romania) and association with risk factors. Vet. Parasitol. 2010, 171, 163-166. [CrossRef]

99. Monteiro, M.F.M.; Ramos, R.A.N.; Calado, A.M.C.; Lima, V.F.S.; Ramos, I.C.D.; Tenorio, R.F.L.; Faustino, M.A.D.; Alves, L.C. Gastrointestinal parasites of cats in Brazil: Frequency and zoonotic risk. Rev. Bras. Parasitol. Vet. 2016, 25, 254-257. [CrossRef]

100. Njuguna, A.N.; Kagira, J.M.; Karanja, S.M.; Ngotho, M.; Mutharia, L.; Maina, N.W. Prevalence of Toxoplasma gondii and other gastrointestinal parasites in domestic cats from households in Thika region, Kenya. Biomed Res. Int. 2017. [CrossRef]

101. Rojekittikhun, W.; Chaisiri, K.; Mahittikorn, A.; Pubampen, S.; Sa-nguankiat, S.; Kusolsuk, T.; Maipanich, W.; Udonsom, R.; Mori, H. Gastrointestinal parasites of dogs and cats in a refuge in Nakhon Nayok, Thailand. Southeast Asian J. Trop. Med. Public Health 2014, 45, 31-39. [PubMed] 
102. Sedionoto, B.; Anamnart, W. Prevalence of hookworm infection and strongyloidiasis in cats and potential risk factor of human diseases. E3S Web of Conf. 2018, 31, 06002. [CrossRef]

103. Takeuchi-Storm, N.; Mejer, H.; Al-Sabi, M.N.S.; Olsen, C.S.; Thamsborg, S.M.; Enemark, H.L. Gastrointestinal parasites of cats in Denmark assessed by necropsy and concentration momaster technique. Vet. Parasitol. 2015, 214, 327-332. [CrossRef] [PubMed]

104. Ekanayake, D.K.; Arulkanthan, A.; Horadagoda, N.U.; Sanjeevani, G.K.M.; Kieft, R.; Gunatilake, S.; Dittus, W.P.J. Prevalence of cryptosporidium and other enteric parasites among wild non-human primates in Polonnaruwa, Sri Lanka. Am. J. Trop. Med. Hyg. 2006, 74, 322-329. [CrossRef] [PubMed]

105. Gillespie, T.R.; Greiner, E.C.; Chapman, C.A. Gastrointestinal parasites of the Guenons of Western Uganda. J. Parasitol. 2004, 90, 1356-1360. [CrossRef] [PubMed]

106. Gillespie, T.R.; Greiner, E.C.; Chapman, C.A. Gastrointestinal parasites of the Colobus monkeys of Uganda. J. Parasitol. 2005, 91, 569-573. [CrossRef] [PubMed]

107. Gillespie, T.R.; Lonsdorf, E.V.; Canfield, E.P.; Meyer, D.J.; Nadler, Y.; Raphael, J.; Pusey, A.E.; Pond, J.; Pauley, J.; Mlengeya, T.; et al. Demographic and ecological effects on patterns of parasitism in Eastern chimpanzees (Pan troglodytes schweinfurthii) in Gombe national park, Tanzania. Am. J. Phys. Anthropol. 2010, 143, 534-544. [CrossRef] [PubMed]

108. Gotoh, S. Regional differences in the infection of wild Japanese macaques by gastrointestinal helminth parasites. Primates 2000, 41, 291-298. [CrossRef]

109. Hodder, S.A.M.; Chapman, C.A. Do nematode infections of red colobus (Procolobus rufomitratus) and black-and-white colobus (Colobus guereza) on humanized forest edges differ from those on nonhumanized forest edges? Int. J. Primatol. 2012, 33, 845-859. [CrossRef]

110. Klaus, A.; Zimmermann, E.; Roper, K.M.; Radespiel, U.; Nathan, S.; Goossens, B.; Strube, C. Co-infection patterns of intestinal parasites in arboreal primates (Proboscis monkeys, Nasalis larvatus) in Borneo. Int. J. Parasitol. Parasit. Wildl. 2017, 6, 320-329. [CrossRef]

111. Knezevich, M. Geophagy as a therapeutic mediator of endoparasitism in a free-ranging group of Rhesus macaques (Macaca mulatta). Am. J. Primatol. 1998, 44, 71-82. [CrossRef]

112. Kooriyama, T.; Hasegawa, H.; Shimozuru, M.; Tsubota, T.; Nishida, T.; Iwaki, T. Parasitology of five primates in Mahale mountains national park, Tanzania. Primates 2012, 53, 365-375. [CrossRef] [PubMed]

113. Kouassi, R.Y.W.; McGraw, S.W.; Yao, P.K.; Abou-Bacar, A.; Brunet, J.; Pesson, B.; Bonfoh, B.; N'Goran, E.K.; Candolfi, E. Diversity and prevalence of gastrointestinal parasites in seven non-human primates of the Tai national park, Cote D'ivoire. Parasite 2015, 22. [CrossRef] [PubMed]

114. Kumar, S.; Sundararaj, P.; Kumara, H.N.; Pal, A.; Santhosh, K.; Vinoth, S. Prevalence of gastrointestinal parasites in bonnet macaque and possible consequences of their unmanaged relocations. PLoS ONE 2018, 13. [CrossRef] [PubMed]

115. Kuze, N.; Kanamori, T.; Malim, T.P.; Bernard, H.; Zamma, K.; Kooriyama, T.; Morimoto, A.; Hasegawa, H. Parasites found from the feces of Bornean orangutans in Danum Valley, Sabah, Malaysia, with a redescription of Pongobius hugoti and the description of a new species of Pongobius (nematoda: Oxyuridae). J. Parasitol. 2010, 96, 954-960. [CrossRef] [PubMed]

116. Labes, E.M.; Wijayanti, N.; Deplazes, P.; Mathis, A. Genetic characterization of Strongyloides spp. From captive, semi-captive and wild Bornean orangutans (Pongo pygmaeus) in central and east Kalimantan, Borneo, Indonesia. Parasitology 2011, 138, 1417-1422. [CrossRef]

117. Legesse, M.; Erko, B. Zoonotic intestinal parasites in Papio anubis (Baboon) and Cercopithecus aethiops (Vervet) from four localities in Ethiopia. Acta Trop. 2004, 90, 231-236. [CrossRef] [PubMed]

118. Levecke, B.; Dorny, P.; Geurden, T.; Vercammen, F.; Vercruysse, J. Gastrointestinal protozoa in non-human primates of four zoological gardens in Belgium. Vet. Parasitol. 2007, 148, 236-246. [CrossRef]

119. Mafuyai, H.B.; Barshep, Y.; Audu, B.S.; Kumbak, D.; Ojobe, T.O. Baboons as potential reservoirs of zoonotic gastrointestinal parasite infections at Yankari National Park, Nigeria. Afr. Health Sci. 2013, 13, $252-254$. [CrossRef]

120. Maldonado-Lopez, S.; Maldonado-Lopez, Y.; Ch, A.G.T.; Cuevas-Reyes, P.; Stoner, K.E. Patterns of infection by intestinal parasites in sympatric howler monkey (Alouatta palliata) and spider monkey (Ateles geoffroyi) populations in a tropical dry forest in Costa Rica. Primates 2014, 55, 383-392. [CrossRef] 
121. Martin-Solano, S.; Carrillo-Bilbao, G.A.; Ramirez, W.; Celi-Erazo, M.; Huynen, M.C.; Levecke, B.; Benitez-Ortiz, W.; Losson, B. Gastrointestinal parasites in captive and free-ranging Cebus albifrons in the Western Amazon, Ecuador. Int. J. Parasitol. Parasit. Wil. 2017, 6, 209-218. [CrossRef] [PubMed]

122. Matsubayashi, K.; Gotoh, S.; Kawamoto, Y.; Watanabe, T.; Nozawa, K.; Takasaka, M.; Narita, T.; Griffiths, O.; Stanley, M.A. Clinical examinations on crab-eating macaques in Mauritius. Primates 1992, 33, 281-288. [CrossRef]

123. McLennan, M.R.; Hasegawa, H.; Bardi, M.; Huffman, M.A. Gastrointestinal parasite infections and self-medication in wild chimpanzees surviving in degraded forest fragments within an agricultural landscape mosaic in Uganda. PLoS ONE 2017, 12. [CrossRef] [PubMed]

124. Muehlenbein, M.P. Parasitological analyses of the male chimpanzees (Pan troglodytes schweinfurthii) at Ngogo, Kibale national park, Uganda. Am. J. Primatol. 2005, 65, 167-179. [CrossRef] [PubMed]

125. Munene, E.; Otsyula, M.; Mbaabu, D.A.N.; Mutahi, W.T.; Muriuki, S.M.K.; Muchemi, G.M. Helminth and protozoan gastrointestinal tract parasites in captive and wild-trapped African non-human primates. Vet. Parasitol. 1998, 78, 195-201. [CrossRef]

126. Muriuki, S.M.K.; Murugu, R.K.; Munene, E.; Karere, G.M.; Chai, D.C. Some gastro-intestinal parasites of zoonotic (public health) importance commonly observed in old world non-human primates in Kenya. Acta Trop. 1998, 71, 73-82. [CrossRef]

127. Parr, N.A.; Fedigan, L.M.; Kutz, S.J. A coprological survey of parasites in white-faced Capuchins (Cebus capucinus) from sector Santa Rosa, acg, Costa Rica. Folia Primatol. 2013, 84, 102-114. [CrossRef]

128. Petrasova, J.; Modry, D.; Huffman, M.A.; Mapua, M.I.; Bobakova, L.; Mazoch, V.; Singh, J.; Kaur, T.; Petrzelkova, K.J. Gastrointestinal parasites of Indigenous and introduced primate species of Rubondo island national park, Tanzania. Int. J. Primatol. 2010, 31, 920-936. [CrossRef]

129. Petrzelkova, K.J.; Hasegawa, H.; Appleton, C.C.; Huffman, M.A.; Archer, C.E.; Moscovice, L.R.; Mapua, M.I.; Singh, J.; Kaur, T. Gastrointestinal parasites of the chimpanzee population introduced onto Rubondo island national park, Tanzania. Am. J. Primatol. 2010, 72, 307-316. [CrossRef]

130. Phillips, K.A.; Haas, M.E.; Grafton, B.W.; Yrivarren, M. Survey of the gastrointestinal parasites of the primate community at Tambopata national reserve, Peru. J. Zool. 2004, 264, 149-151. [CrossRef]

131. Pouillevet, H.; Dibakou, S.E.; Ngoubangoye, B.; Poirotte, C.; Charpentier, M.J.E. A comparative study of four methods for the detection of nematode eggs and large protozoan cysts in mandrill faecal material. Folia Primatol. 2017, 88, 344-357. [CrossRef] [PubMed]

132. Pourrut, X.; Diffo, J.L.D.; Somo, R.M.; Bilong, C.F.B.; Delaportee, E.; LeBreton, M.; Gonzalez, J.P. Prevalence of gastrointestinal parasites in primate bushmeat and pets in Cameroon. Vet. Parasitol. 2011, 175, 187-191. [CrossRef] [PubMed]

133. Ryan, S.J.; Brashares, J.S.; Walsh, C.; Milbers, K.; Kilroy, C.; Chapman, C.A. A survey of gastrointestinal parasites of olive baboons (Papio anubis) in human settlement areas of Mole national park, Ghana. J. Parasitol. 2012, 98, 885-888. [CrossRef] [PubMed]

134. Solorzano-Garcia, B.; de Leon, G.P.P. Helminth parasites of howler and spider monkeys in Mexico: Insights into molecular diagnostic methods and their importance for zoonotic diseases and host conservation. Int. J. Parasitol. Parasit. Wil. 2017, 6, 76-84. [CrossRef] [PubMed]

135. Thanchomnang, T.; Intapan, P.M.; Sanpool, O.; Rodpai, R.; Sadaow, L.; Phosuk, I.; Somboonpatarakun, C.; Laymanivong, S.; Tourtip, S.; Maleewong, W. First molecular identification of Strongyloides fuelleborni in long-tailed macaques in Thailand and Lao People's Democratic Republic reveals considerable genetic diversity. J. Helminthol. 2018, 1-8. [CrossRef] [PubMed]

136. Tiwari, S.; Reddy, D.M.; Pradheeps, M.; Sreenivasamurthy, G.S.; Umapathy, G. Prevalence and co-occurrence of gastrointestinal parasites in Nilgiri Langur (Trachypithecus johnii) of fragmented landscape in Anamalai hills, Western Ghats, India. Curr. Sci. 2017, 113, 2194-2200. [CrossRef]

137. Wenz, A.; Heymann, E.W.; Petney, T.N.; Taraschewski, H.F. The influence of human settlements on the parasite community in two species of Peruvian Tamarin. Parasitology 2010, 137, 675-684. [CrossRef] [PubMed]

138. Wren, B.T.; Gillespie, T.R.; Camp, J.W.; Remis, M.J. Helminths of vervet monkeys, Chlorocebus aethiops, from Loskop Dam nature reserve, South Africa. Comp. Parasitol. 2015, 82, 101-108. [CrossRef]

139. Wren, B.T.; Remis, M.J.; Camp, J.W.; Gillespie, T.R. Number of grooming partners is associated with hookworm infection in wild vervet monkeys (Chlorocebus aethiops). Folia Primatol. 2016, 87, 168-179. [CrossRef] [PubMed] 
140. Frias, L.; Stark, D.J.; Lynn, M.S.; Nathan, S.K.; Goossens, B.; Okamoto, M.; MacIntosh, A.J.J. Lurking in the dark: Cryptic strongyloides in a Bornean slow loris. Int. J. Parasitol. Parasites Wil. 2018, 7, 141-146. [CrossRef]

141. Koinari, M.; Karl, S.; Ryan, U.; Lymbery, A.J. Infection levels of gastrointestinal parasites in sheep and goats in Papua New Guinea. J. Helminthol. 2013, 87, 409-415. [CrossRef] [PubMed]

142. MacGlaflin, C.E.; Zajac, A.M.; Rego, K.A.; Petersson, K.H. Effect of vitamin E supplementation on naturally acquired parasitic infection in lambs. Vet. Parasitol. 2011, 175, 300-305. [CrossRef]

143. McManus, C.; Louvandini, H.; Paiva, S.R.; de Oliveira, A.A.; Azevedo, H.C.; de Melo, C.B. Genetic factors of sheep affecting gastrointestinal parasite infections in the Distrito Federal, Brazil. Vet. Parasitol. 2009, 166, 308-313. [CrossRef] [PubMed]

144. Andreassen, P.N.S.; Schmidt, N.M.; Kapel, C.M.O.; Christensen, M.U.; Sittler, B.; Gilg, O.; Enemark, H.L.; Al-Sabi, M.N.S. Gastrointestinal parasites of two populations of arctic foxes (Vulpes lagopus) from North-East Greenland. Polar Res. 2017, 36. [CrossRef]

145. Dalimi, A.; Sattari, A.; Motamedi, G. A study on intestinal helminthes of dogs, foxes and jackals in the western part of Iran. Vet. Parasitol. 2006, 142, 129-133. [CrossRef] [PubMed]

146. Hernandez-Camacho, N.; Pineda-Lopez, R.; Lopez-Gonzalez, C.A.; Jones, R.W. Nematodes parasites of the gray fox (Urocyon cinereoargenteus schreber, 1775) in the seasonally dry tropical highlands of central Mexico. Parasitol. Res. 2011, 108, 1425-1429. [CrossRef] [PubMed]

147. Miterpakova, M.; Hurnikova, Z.; Antolova, D.; Dubinsky, P. Endoparasites of red fox (Vulpes vulpes) in the Slovak Republic with the emphasis on zoonotic species Echinococcus multilocularis and Trichinella spp. Helminthologia 2009, 46, 73-79. [CrossRef]

148. Da Silva, S.R.M.; Maldonade, I.R.; Ginani, V.C.; Lima, S.A.; Mendes, V.S.; Azevedo, M.L.X.; Gurgel-Gonçalves, R.; Machado, E.R. Detection of intestinal parasites on field-grown strawberries in the federal district of Brazil. Rev. Soc. Bras. Med. Trop. 2014, 47, 801-805. [CrossRef]

149. Aghaindum, A.G.; Landry, F.K.A. Dissemination of the resistant forms of intestinal worms in the marshy areas of the city of Yaounde (Cameroon): Importance of some abiotic factors of the medium. Appl. Water Sci. 2019, 9. [CrossRef]

150. Hilles, A.H.; Al Hindi, A.I.; Abu Safieh, Y.A. Assessment of parasitic pollution in the coastal seawater of Gaza city. J. Environ. Health Sci. Eng. 2014, 12. [CrossRef] [PubMed]

151. Bakir, B.; Tanyuksel, M.; Saylam, F.; Tanriverdi, S.; Araz, R.E.; Hacim, A.K.; Hasde, M. Investigation of waterborne parasites in drinking water sources of Ankara, Turkey. J. Microbiol. 2003, 41, 148-151.

152. El-Badry, A.A.; Hamdy, D.A.; El Wahab, W.M.A. Strongyloides stercoralis larvae found for the first time in tap water using a novel culture method. J. Parasitol. Res. 2018, 117, 3775-3780. [CrossRef] [PubMed]

153. Freitas, D.A.; Cabral, J.J.S.P.; Rocha, F.J.S.; Paiva, A.L.R.; Sens, M.L.; Veras, T.B. Cryptosporidium spp. and Giardia spp. removal by bank filtration at Beberibe River, Brazil. J. River Res. App. 2017, 33, 1079-1087. [CrossRef]

154. Klotz, S.A.; Normand, R.E.; Kalinsky, R.G. "Through a drinking glass and what was found there": Pseudocontamination of a hospital's drinking water. J. Infect. Control Hosp. Epidemiol. 1992, 8, 477-481.

155. Zeehaida, M.; Zairi, N.Z.; Rahmah, N.; Maimunah, A.; Madihah, B. Strongyloides stercoralis in common vegetables and herbs in Kota Bharu, Keletan, Malaysia. J. Trop. Biomed. 2011, 28, 188-193.

156. Luz, J.G.G.; Barbosa, M.V.; de Carvalho, A.G.; Resende, S.D.; Dias, J.V.L.; Martins, H.R. Contamination by intestinal parasites in vegetables marketed in an area of Jequitinhonha Valley, Minas Gerais, Brazil. Rev. Nutricao-Braz. J. Nutr. 2017, 30, 127-136. [CrossRef]

157. Maikai, B.V.; Elisha, I.A.; Baba-Onoja, E.B.T. Contamination of vegetables sold in markets with helminth eggs in Zaria metropolis, Kaduna State, Nigeria. Food Control 2012, 28, 345-348. [CrossRef]

158. Madadi, M. Parasitic contamination in the table vegetables planted in Shiraz plain, Iran. Pak. J. Sci. Ind. Res. 2010, 53, 42-45.

159. Berentsen, A.R.; Becker, M.S.; Stockdale-Walden, H.; Matandiko, W.; McRobb, R.; Dunbar, M.R. Survey of gastrointestinal parasite infection in African lion (Panthera leo), African wild dog (Lycaon pictus) and spotted hyaena (Crocuta crocuta) in the Luangwa valley, Zambia. Afr. Zool. 2012, 47, 363-368. [CrossRef]

160. Bista, D.; Shrestha, S.; Kunwar, A.J.; Acharya, S.; Jnawali, S.R.; Acharya, K.P. Status of gastrointestinal parasites in red panda of Nepal. PeerJ 2017, 2017. [CrossRef] 
161. Cardia, D.F.F.; Camossi, L.G.; Fornazari, F.; Babboni, S.D.; Teixeira, C.R.; Bresciani, K.D.S. First report of Strongyloides sp. (nematoda, Strongyloididae) in Lutreolina crassicaudata (didelphimorphia: Didelphidae). Braz. J. Biol. 2016, 76, 884-887. [CrossRef] [PubMed]

162. Cordon, G.P.; Prados, A.H.; Romero, D.; Moreno, M.S.; Pontes, A.; Osuna, A.; Rosales, M.J. Intestinal parasitism in the animals of the zoological garden "Pena Escrita" (Almunecar, Spain). Vet. Parasitol. 2008, 156, 302-309. [CrossRef] [PubMed]

163. González, P.; Carbonell, E.; Urios, V.; Rozhnov, V.V. Coprology of Panthera tigris altaica and Felis bengalensis euptilurus from the Russian far East. J. Parasitol. 2007, 93, 948-950. [CrossRef] [PubMed]

164. Gupta, A.; Singh, N.K.; Singh, H.; Rath, S.S. Assessment of risk factors associated with prevalence of gastrointestinal helminths in buffaloes from Punjab State, India. Buffalo Bull. 2018, 37, 279-290.

165. Hallinger, M.J.; Taubert, A.; Hermosilla, C.; Mutschmann, F. Occurrence of health-compromising protozoan and helminth infections in tortoises kept as pet animals in Germany. Parasites Vectors 2018, 11. [CrossRef]

166. Hasegawa, H.; Ota, H. Parasitic Helminths Found from Polypedates leucomystax (Amphibia: Rhacophoridae) on Miyakojima Island, Ryukyu Archipelago, Japan. Curr. Herpetol. 2017, 36, 1-10. [CrossRef]

167. Hermosilla, C.; Kleinertz, S.; Silva, L.M.R.; Hirzmann, J.; Huber, D.; Kusak, J.; Taubert, A. Protozoan and helminth parasite fauna of free-living Croatian wild wolves (Canis lupus) analyzed by scat collection. Vet. Parasitol. 2017, 233, 14-19. [CrossRef]

168. Hu, X.L.; Liu, G.; Wei, Y.T.; Wang, Y.H.; Zhang, T.X.; Yang, S.; Hu, D.F.; Liu, S.Q. Regional and seasonal effects on the gastrointestinal parasitism of captive forest musk deer. Acta Tropica 2018, 177, 1-8. [CrossRef]

169. Huang, W.; Zhou, L.Z.; Zhao, N.N. Temporal-spatial patterns of intestinal parasites of the Hooded Crane (Grus monacha) wintering in lakes of the middle and lower Yangtze River floodplain. Avian Res. 2014, 5. [CrossRef]

170. Kumba, F.F.; Katjivena, H.; Kauta, G.; Lutaaya, E. Seasonal evolution of faecal egg output by gastrointestinal worms in goats on communal farms in eastern Namibia. Onderstepoort J. Vet. Res. 2003, 70, 265-271. [CrossRef]

171. Mizgajska-Wiktor, H.; Jarosz, W. Potential risk of zoonotic infections in recreational areas visited by Sus scrofa and Vulpes vulpes. Case study-Wolin Island, Poland. Wiad. Parazytol. 2010, 56, $243-251$.

172. Mukul-Yerves, J.M.; Zapata-Escobedo, M.D.; Montes-Perez, R.C.; Rodriguez-Vivas, R.I.; Torres-Acosta, J.F. Gastrointestinal and ectoparasites in wildlife-ungulates under captive and free-living conditions in the Mexican tropic. Rev. Mex. Cienc. Pecu. 2014, 5, 459-469.

173. Oja, R.; Velstrom, K.; Moks, E.; Jokelainen, P.; Lassen, B. How does supplementary feeding affect endoparasite infection in wild boar? Parasitol. Res. 2017, 116, 2131-2137. [CrossRef] [PubMed]

174. Ojeda-Robertos, N.; Torres-Chable, O.M.; Peralta-Torres, J.; Luna-Palomera, C.; Aguilar-Cabrales, A.; Chay-Canul, A.; Gonzalez-Garduno, R.; Machain-Williams, C.; Camara-Sarmiento, R. Study of gastrointestinal parasites in water buffalo (Bubalus bubalis) reared under Mexican humid tropical conditions. Trop. Anim. Health Prod. 2017, 49, 613-618. [CrossRef] [PubMed]

175. Perera, A.; Maia, J.; Jorge, F.; Harris, D.J. Molecular screening of nematodes in lacertid lizards from the Iberian Peninsula and Balearic Islands using 18s rRNA sequences. J. Helminthol. 2013, 87, 189-194. [CrossRef] [PubMed]

176. Pilarczyk, B.; Tomza-Marciniak, A.; Udala, J.; Kuba, J. The prevalence and control of gastrointestinal nematodes in farmed fallow deer (Dama dama 1.). Vet. Arh. 2015, 85, 415-423.

177. Rahman, M.; Islam, S.; Masuduzzaman, M.; Alam, M.; Chawdhury, M.N.U.; Ferdous, J.; Islam, M.N.; Hassan, M.M.; Hossain, M.A.; Islam, A. Prevalence and diversity of gastrointestinal helminths in free-ranging Asian house shrew (Suncus murinus) in Bangladesh. Vet. World 2018, 11, 549-556. [CrossRef] [PubMed]

178. Rinaldi, L.; Musella, V.; Veneziano, V.; Condoleo, R.U.; Cringoli, G. Helmintic infections in water buffaloes on Italian farms: A spatial analysis. Geospat. Health 2009, 3, 233-239. [CrossRef] [PubMed]

179. Rosalino, L.M.; Torres, J.; Santos-Reis, M. A survey of helminth infection in Eurasian badgers (Meles meles) in relation to their foraging behaviour in a mediterranean environment in Southwest Portugal. Eur. J. Wildl. Res. 2006, 52, 202-206. [CrossRef]

180. Turni, C.; Smales, L.R. Parasites of the bridled nailtail wallaby (Onychogalea fraenata) (marsupialia : Macropodidae). Wildl. Res. 2001, 28, 403-411. [CrossRef]

181. Turner, W.C.; Getz, W.M. Seasonal and demographic factors influencing gastrointestinal parasitism in ungulates of Etosha national park. J. Wildl. Dis. 2010, 46, 1108-1119. [CrossRef] [PubMed] 
182. Turner, W.C.; Cizauskas, C.A.; Getz, W.M. Variation in faecal water content may confound estimates of gastro-intestinal parasite intensity in wild African herbivores. J. Helminthol. 2010, 84, 99-105. [CrossRef] [PubMed]

183. VanderWaal, K.; Omondi, G.P.; Obanda, V. Mixed-host aggregations and helminth parasite sharing in an East African wildlife-livestock system. Vet. Parasitol. 2014, 205, 224-232. [CrossRef] [PubMed]

184. Ybañez, R.H.D.; Resuelo, K.J.G.; Kintanar, A.P.M.; Ybañez, A.P. Detection of gastrointestinal parasites in small-scale poultry layer farms in Leyte, Philippines. Vet. World 2018, 11, 1587-1591. [CrossRef] [PubMed]

(C) 2019 by the authors. Licensee MDPI, Basel, Switzerland. This article is an open access article distributed under the terms and conditions of the Creative Commons Attribution (CC BY) license (http://creativecommons.org/licenses/by/4.0/). 